\title{
A Branch-Price-and-Cut Procedure for the Discrete Ordered Median Problem
}

\author{
Samuel Deleplanque \\ Ifsttar, COSYS, ESTAS, Université Lille Nord de France, \\ Martine Labbé \\ Départament d'Informatique, Faculté des Sciences, \\ Université Libre de Bruxelles, \\ Diego Ponce \\ Instituto de Matemáticas de la Universidad de Sevilla (IMUS), \\ Department of Mechanical, Industrial and Aerospace Engineering, Concordia University, \\ Centre interuniversitaire de recherche sur les réseaux d'enterprise, la logistique et le transport (CIRRELT), \\ Justo Puerto \\ Instituto de Matemáticas de la Universidad de Sevilla (IMUS).
}

December 13, 2018

\begin{abstract}
The Discrete Ordered Median Problem (DOMP) is formulated as a set partitioning problem using an exponential number of variables. Each variable corresponds to a set of demand points allocated to the same facility with the information of the sorting position of their corresponding costs. We develop a column generation approach to solve the continuous relaxation of this model. Then, we apply a branch-price-and-cut algorithm to solve small to large sized instances of DOMP in competitive computational time.
\end{abstract}

\section{Introduction}

Logistics is a very active domain in contemporary Operations Research and Location Analysis is one of its main building blocks. In the last years, motivated by the need of applying more flexible models in Logistics, a new family of location models that takes into consideration the order of the costs to be minimized has emerged in the literature.

The ordered weighted average of a vector is equal to the weighted sum of its coordinates after ranking them by non decreasing order (Nickel and Puerto (2005)). When the ordered weighted average is used in a location problem, the vector contains the distances or allocation costs from clients to service facilities. Ordered median location problems were first introduced in networks and continuous spaces by Nickel and Puerto (1999) and Puerto and Fernández (2000), respectively. Later, they were extended to the discrete setting by Nickel (2001) and Boland et al. (2006). The Discrete Ordered Median Problem (DOMP) has been widely studied since the 90's and there is a number of different formulations, solution approaches and applications available in the literature. To cite a few, DOMP has been applied to discrete facility location in Boland et al. (2006); Marín et al. (2009, 2010); Nickel (2001); Puerto (2008); Puerto et al. (2009); to location on networks in Nickel and Puerto (1999); to hub network design problems in Puerto et al. (2011, 2013, 2016); to determine values in cooperative game theory in Perea and Puerto (2013); to combinatorial optimization problems 
with ordering in Fernández et al. (2013, 2014, 2017), and to voting problems in Ponce et al. (2018), etc. The reader is referred to the monographies by Nickel and Puerto (2005) and Puerto and Rodríguez-Chía (2015) for some other applications.

Given a set of clients, a set of candidate locations and assuming that the allocation costs of clients to facilities are known, DOMP consists in choosing $p$ facility locations and assigning each client to a chosen facility with the smallest allocation cost in order to minimize the ordered weighted average of these costs. The ordered weighted average sorts the allocation costs in a non-decreasing sequence and then it performs the scalar product of this so-obtained sorted cost vector with a given vector of weights.

There are several valid formulations for DOMP that exploit specific features of the problem (see e.g. Boland et al. (2006); Marín et al. (2009); Labbé et al. (2017) and the references therein). In Labbé et al. (2017) a new formulation for DOMP has been proposed, based on a set packing approach, that is valid for general cost coefficients. This formulation gives rise to rather tight integrality gaps and was shown to be reasonably efficient to solve medium size instances when embedded in a branch-and-cut $(\mathbf{B} \& \mathbf{C})$ scheme. In this paper we explore a different paradigm for solving DOMP based on an extended formulation using an exponential number of variables and that corresponds to a set partitioning model. Each variable represents a set of couples (client, position). These clients are served by the same facility and their position indicates the place of their allocation cost in the sorted list of allocation costs for the entire considered solution. To handle the exponential number of variables we use a column generation approach that is embedded in a branch-price-and-cut ( $\mathbf{B} \& \mathbf{P} \& \mathbf{C})$ algorithm. A recent similar approach to a different problem can be found in Doulabi et al. (2016). This scheme has never been applied to DOMP and it opens new avenues of research. Therefore, the contribution of this paper is to propose a new perspective in the resolution of DOMP based on formulations with an exponential number of variables and to develop an efficient $\mathbf{B} \& \mathbf{P} \& \mathbf{C}$ algorithm to handle them.

The remainder of this paper is organized as follows. Notation, models and algorithms are presented in Section 2. Subsection 2.2 introduces a new set partitioning formulation for DOMP. This formulation uses an exponential number of variables where each element of the partition is a set of clients that are assigned to the same facility together with their sorted positions. This formulation is theoretically compared with another valid formulation described in Subsection 2.1 and borrowed from Labbé et al. (2017). Subsection 2.3 describes the column generation algorithm that we have designed to overcome the large number of variables in the model. We prove that the pricing subproblem is solvable efficiently in polynomial time by using an ad hoc dynamic programming algorithm. We devote Section 3 to the implementation details of our B\&P\&C algorithm. We develop a GRASP heuristic, in Subsection 3.1, that is used to generate both a promising initial solution and a pool of variables to initialize the column generation routine. We also develop a stabilization routine, based on Pessoa et al. (2010), that reduces considerably the number of iterations of the column generation approach in Subsection 3.2. In addition, Subsection 3.3 is devoted to an additional improvement, namely a preprocessing. The next two subsections, 3.4 and 3.5, present our branching strategies and some families of valid inequalities that will be added to the branch-and-price algorithm. In the last section, namely Section 4, we report on the final computational experiments. We evaluate the performance of the $\mathbf{B} \& \mathbf{P} \& \mathbf{C}$ algorithm and compare it to the compact formulation in Section 2.1. The paper ends with some concluding remarks. 


\section{Problem definition and formulations}

Let $I$ be a set of $n$ points which at the same time represent clients and potential uncapacitated facility locations and let $c_{i j}$ denote the cost for serving client $i$ 's demand from facility $j$.

Given a set $J$ of $p$ open facilities, let $c_{i}(J)$ represent the cheapest cost for allocating client $i$ to a facility in $J$, i.e. $c_{i}(J):=\min _{j \in J} c_{i j}$.

Now let us sort the costs $c_{i}(J), i \in I$ by non-decreasing order of their values. The elements of the resulting vector of ordered costs are denoted by $c^{(k)}(J)$ and satisfy $c^{(1)}(J) \leq \cdots \leq$ $c^{(n)}(J)$. We denote the set of all possible positions $1, \ldots, n$ in this ordered vector by $K$.

Given vector $\lambda=\left(\lambda^{k}\right)_{k \in K}$ satisfying $\lambda^{k} \geq 0, k \in K$, the objective function of DOMP, is defined as

$$
z(J):=\sum_{k \in K} \lambda^{k} c^{(k)}(J)
$$

Recall that this objective function provides a very general paradigm to encompass standard and new location models. For instance, if $\lambda^{1}=\ldots=\lambda^{n}=1$ we obtain the median objective, if $\lambda^{1}=\lambda^{2}=\ldots=\lambda^{n-1}=0, \lambda^{n}=1$ we obtain the center objective, if $\lambda^{1}=\lambda^{2}=\ldots=\lambda^{n-1}=\alpha, \lambda^{n}=1$, where $\alpha=[0,1]$, we obtain a convex combination of median and center objectives (centdian), etc.

The $p$-facility Discrete Ordered Median Problem looks for the subset $J$ of $p$ facilities to open in order to minimize the ordered median function:

$$
\min _{J \subseteq I:|J|=p} z(J) .
$$

Several formulations of DOMP have been proposed in the literature using different types of variables. Among them we mention those based on a combination of the $p$-median and permutation polytopes (Boland et al. (2006)) or on covering approaches by using radius variables (Puerto (2008), Marín et al. (2009, 2010)).

\subsection{An explicit formulation for DOMP: The Weak Order Constraints}

In the following, we recall the Weak Order Constraints formulation, WOC, introduced in Labbé et al. (2017), and that will be the starting point for the developments presented in this paper. This formulation uses two types of binary variables. Variable $y_{j}$ assumes value 1 if facility $j \in I$ is open (i.e. $j \in J$ ) and 0 otherwise. Variable $x_{i j}^{k}$ is equal to 1 if client $i \in I$ is allocated to facility $j \in I$ and the corresponding cost occupies position $k \in K$ in the allocation cost ranking (i.e. $c^{(k)}(J)=c_{i j}$ ). The choice of this formulation is motivated by its good performance in terms of integrality gap (see Labbé et al. (2017)). However, it requests important memory space since it needs $O\left(n^{3}\right)$ binary variables which may become prohibitive for moderate $n$.

We denote the rank of the allocation cost $c_{i j}$ by $r_{i j}$, i.e. $r_{i j}=\ell$ if $c_{i j}$ is the $\ell$-th element in the list of the $\operatorname{costs} c_{i j}$, for all $i, j \in I$, sorted by order of non decreasing values and where ties are broken arbitrarily. For the sake of readability the reader is referred to Example 1 in Section 2.3. The formulation is as follows:

$$
\begin{array}{cc}
\text { (WOC) } \min & \sum_{i \in I} \sum_{j \in I} \sum_{k \in K} \lambda^{k} c_{i j} x_{i j}^{k} \\
\text { s.t. } & \sum_{j \in I} \sum_{k \in K} x_{i j}^{k}=1
\end{array} \quad i \in I
$$




$$
\begin{aligned}
& \sum_{i \in I} \sum_{j \in I} x_{i j}^{k}=1 \quad k \in K \\
& \sum_{k \in K} x_{i j}^{k} \leq y_{j} \quad i, j \in I \\
& \sum_{j \in I} y_{j}=p \\
& \sum_{i \in I} \sum_{j \in I}\left(\sum_{i^{\prime} \in I} \sum_{\substack{j^{\prime} \in I: \\
r_{i^{\prime} j^{\prime}} \leq r_{i j}}} x_{i^{\prime} j^{\prime}}^{k}+\sum_{i^{\prime} \in I} \sum_{\substack{j^{\prime} \in I: \\
r_{i^{\prime} j^{\prime}} \leq r_{i j}}} x_{i^{\prime} j^{\prime}}^{k-1}\right) \leq n^{2} \quad k \in K, k \neq 1 \\
& x_{i j}^{k}, y_{j} \in\{0,1\} \quad i, j \in I, k \in K .
\end{aligned}
$$

By means of (3) we ensure that each location is served by exactly one facility. In the same way, in each position there must be exactly one allocation cost (4). Constraints (5) translate the fact that a client can be allocated to a facility only if this facility is open and that the allocation cost of a client to a facility can be placed in at most one position. The equality constraint (6) implies that there are exactly $p$ open facilities.

Constraints (7), called weak order constraints, ensure that if client $i$ is allocated to facility $j$ and the corresponding costs $c_{i j}$ occupies the $k$-th position in the cost ranking of the solution then in the $(k-1)$-th position there must be a smaller allocation cost. This property is enforced by the coefficients of each variable in the inequality. In each constraint there are two different positions, $k$ and $k-1$, so that, by (4), only two variables must take value one and all the others will be equal to zero. If we do not take into account the variables assuming the value zero and we assume that the variables with value one for positions $k$ and $k-1$ correspond to allocation pairs in sorted position $s$ and $t$, respectively, the inequality reduces to the following expression: $\left(n^{2}-(s-1)\right) x_{i_{s} j_{s}}^{k}+t x_{i_{i} j_{t}}^{k-1} \leq n^{2}$, which is valid if and only if $t<s$. Finally, the variables are binary, see $(8)$.

$W O C$ can be reinforced by adding the following valid inequalities:

$$
\sum_{i^{\prime} \in I} \sum_{\substack{j^{\prime} \in I: \\ r_{i^{\prime} j^{\prime}} \leq r_{i j}}} x_{i^{\prime} j^{\prime}}^{k}+\sum_{i^{\prime} \in I} \sum_{\substack{j^{\prime} \in I: \\ r_{i^{\prime} j^{\prime}} \geq r_{i j}}} x_{i^{\prime} j^{\prime}}^{k-1} \leq 1, i, j \in I, k \in K, k \neq 1 .
$$

Observe that constraints (7) are the aggregation over $i, j \in I$ of inequalities (9). These inequalities are the so called strong order constraints, See Labbé et al. (2017) for a detailed explanation.

\section{$2.2 \quad$ A set partitioning formulation}

From a linear programming relaxation point of view the above formulation is not the strongest one but it provides a good compromise between the number of required constraints and the quality of its linear relaxation bound, see Labbé et al. (2017). Further, it allows to solve to optimality problems of moderate size. One of its drawbacks is the use of a cubic number of variables, which can be prohibitive for large $n$. A second important problem of most known formulations for DOMP is their high degree of symmetry in case of allocation costs $c_{i j}$ or weighted $\left(\lambda^{k}\right)$ with many ties.

The reasons above motivate the introduction of a new formulation based on a different rationale. We observe that a solution for DOMP is a partition of the clients together with their positions in the sorted vector of costs so that each subset of clients in the partition is allocated to the same facility. 
Let us consider sets of couples $(i, k)$ where the first component refers to a client $i$ and the second to a position $k$, namely $S=\{(i, k)$ : for some $i \in I, k \in K\}$. Further, we denote by $\mathcal{P}(I \times K)$ the family of all sets $S$ for which all first (resp. second) coordinates of its couples are different.

Associated with each set $S$ and facility $j$, we define a variable $y_{S}^{j}$ equal to 1 if the set $S$ is part of a feasible solution $\left((i, k) \in S\right.$ iff $\left.x_{i j}^{k}=1\right)$; and 0 otherwise.

Let $S$ be the set of couples whose first coordinate corresponds to the clients allocated to a given facility $j$ of a feasible solution. The positions of these clients in the solution, i.e. the second coordinates of couples in $S$ must be compatible with the ranking of all the allocation costs involved in the solution. Hence, they must, in particular, be compatible with the ranking of the $\operatorname{costs} c_{i j}$ of the clients $i$ allocated to $j$. This implies that for facility $j \in J$ we only need to consider couple subsets $\mathrm{S}$ belonging to $\mathcal{S}(j)=\left\{S \in \mathcal{P}(I \times K): c_{i j} \leq c_{i^{\prime} j}\right.$ for all $(i, k),\left(i^{\prime}, k^{\prime}\right) \in S$ and $\left.k<k^{\prime}\right\}$.

Since in any feasible solution, each client $i$ must be allocated to a unique facility $j$ and its allocation cost must occupy a unique position $k$ in the sorted list, the following relationship holds:

$$
x_{i j}^{k}=\sum_{S \in \mathcal{S}(j):(i, k) \in S} y_{S}^{j}, i, j \in I, k \in K .
$$

Next, we can evaluate the $\operatorname{cost} c_{S}^{j}$ induced by the set $S$ provided that its clients are assigned to facility $j$ in a feasible solution:

$$
c_{S}^{j}=\sum_{(i, k) \in S} \lambda^{k} c_{i j}
$$

To simplify the presentation in the following we denote by $(i, \cdot)$ any couple whose first entry is $i$ regardless of the value of the second entry. Analogously, $(\cdot, k)$ denotes any couple whose second entry is $k$ regardless of the value of the first entry.

The following valid formulation uses variables $y_{S}^{j}$ and constitutes our Master Problem $(M P)$.

$$
\begin{aligned}
& \text { (MP) } \min \quad \sum_{j \in I} \sum_{S \in \mathcal{S}(j)} c_{S}^{j} y_{S}^{j} \\
& \text { s.t. } \quad \sum_{j \in I} \sum_{\substack{S \in \mathcal{S}(j): \\
(i, \cdot) \in S}} y_{S}^{j}=1 \quad i \in I \\
& \sum_{j \in I} \sum_{\substack{S \in \mathcal{S}(j): \\
(\cdot, k) \in S}} y_{S}^{j}=1 \quad k \in K \\
& \sum_{S \in \mathcal{S}(j)} y_{S}^{j} \leq 1 \quad j \in I \\
& \sum_{j \in I} \sum_{S \in \mathcal{S}(j)} y_{S}^{j} \leq p \\
& \sum_{i=1}^{n} \sum_{j=1}^{n}\left(\sum_{\substack{S \in \mathcal{S}(j): \\
\left(i^{\prime}, k\right) \in S \\
r_{i^{\prime} j^{\prime} \leq r_{i j}}}} y_{S}^{j^{\prime}}+\sum_{\substack{S \in \mathcal{S}(j): \\
\left(i^{\prime}, k-1\right) \in S \\
r_{i^{\prime} j^{\prime}} \geq r_{i j}}} y_{S}^{j^{\prime}}\right) \leq n^{2} \quad k \in K, k \neq 1
\end{aligned}
$$




$$
y_{S}^{j} \in\{0,1\} \quad S \in \mathcal{S}(j), j \in I \text {. }
$$

The objective function (12) accounts for the sorted weighted cost of any feasible solution. Constraints (13) ensure that each client appears in exactly one set $S$. Constraints (14) ensure that each position is taken by exactly one client appearing in one set $S$. Constraints (15) guarantees that each facility $j$ serves at most one set $S$ of clients. Inequality (16) states that at most $p$ facilities will be opened. By the following family of inequalities (17) we enforce the correct sorting of the costs in any feasible solution. Finally, the variables are binary.

One can relate $M P$ and $W O C$. First, remark that for a given facility $j$, there is at most one cost $c_{i j}$ which occupies a given position $k$. Hence, the following constraints are valid for $W O C$ :

$$
\sum_{i \in I} x_{i j}^{k} \leq y_{j}, j \in I, k \in K
$$

Let $W O C+$ denote the formulation given by (2)-(8) and (19) and consider the DantzigWolfe reformulation of $W O C+$ in which constraints (5), (19) and (8) constitute the subproblem. The subproblem can be decomposed by facility.

On the one hand, the feasible points of the subproblem of a facility $j$ correspond one to one to the sets $S \in \mathcal{P}(I \times K)$. Hence, this Dantzig-Wolfe reformulation of $W O C+$ is given by the above master problem in which we consider variables $y_{S}^{j}$ for all $S \in \mathcal{P}(I \times K)$ (instead of only $S \in \mathcal{S}(j))$. More precisely, the variables of $W O C+$ are related to the variables $y_{S}^{j}$ through the following two equations:

$$
x_{i j}^{k}=\sum_{S \in \mathcal{P}(I \times K):(i, k) \in S} y_{S}^{j} \quad i, j \in I, k \in K
$$

and

$$
y_{j}=\sum_{S \in \mathcal{P}(I \times K)} y_{S}^{j} \quad j \in I .
$$

Moreover, constraints (13) correspond to constraints (3), constraints (14) to (4), constraints (16) to (6), and constraints (17) to (7). Finally, constraints (15) constitute the "convexity" constraints for the subproblems.

On the other hand, it is easy to see that the polyhedron of each subproblem, defined by constraints (5) and (19) together with $x_{i j}^{k} \geq 0$ and $y_{j} \leq 1$, is integer. This implies that the linear relaxations of $W O C+$ and $M P$ in which all sets $S \in \mathcal{P}(I \times K)$ are considered provide the same bound. By restricting the subsets $S$ to be considered for each facility $j$ to belong to $\mathcal{S}(j)$, our formulation $M P$ provides thus a stronger model. The computational experiments presented in Subsection 4.3 show that there exist instances for which the linear relaxation of $M P$ provides a strictly better (higher) lower bound than the linear relaxation of WOC.

Formulation $M P$ can be strengthened by adding valid inequalities borrowed from $W O C$. Indeed, one can translate valid inequalities (9) in terms of the $y_{S}^{j}$ variables so that they can be used in the set partition formulation of DOMP. The translation of (9) results in:

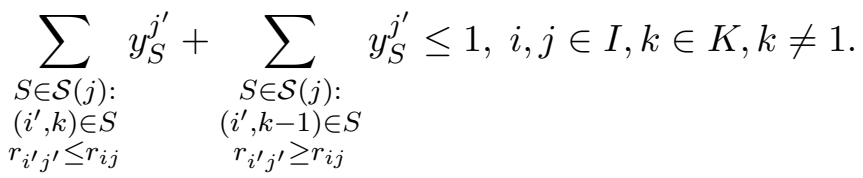




\subsection{Column generation to solve the linear relaxation of $M P$ (LRMP)}

Since the number of variables in $M P$ is too large to be handled directly, in this section, we describe a column generation approach to solve it.

Let $(\alpha, \beta, \gamma, \delta, \epsilon)$ be the dual variables associated, respectively, to constraints (13), (14), (15), (16) and (17). The dual problem DP of LRMP is

$$
\begin{aligned}
& \text { (DP) } \max \quad \sum_{i \in I} \alpha_{i}+\sum_{k \in K} \beta_{k}-\sum_{j \in I} \gamma_{j}-p \delta-\sum_{\substack{k \in K \\
k \neq 1}} n^{2} \epsilon_{k} \\
& \text { s.t. } \quad \sum_{\substack{i \in I: \\
(i, \cdot) \in S}} \alpha_{i}+\sum_{\substack{k \in K: \\
(\cdot, k) \in S}} \beta_{k}-\gamma_{j}-\delta-\sum_{i^{\prime} \in I} \sum_{j^{\prime} \in I}\left(\sum_{\substack{(i, k) \in S: \\
r^{\prime} j^{\prime} \geq r_{i j} \\
k \neq 1}} \epsilon_{k}+\sum_{\begin{array}{c}
(, k) \in S: \\
r_{i}^{\prime} j^{\prime} \leq r_{i j} \\
k \neq n
\end{array}} \epsilon_{k+1}\right) \leq c_{S}^{j} \quad j \in I, S \in \mathcal{S}(j) \\
& \delta, \gamma_{j}, \epsilon_{k} \geq 0 \quad j \in I, k \in K, k \neq 1 .
\end{aligned}
$$

In order to apply the column generation procedure, let us assume that we are given a set of columns that define a restricted Master Problem, and denote its linear relaxation by ReLRMP. This problem is solved to optimality and $\left(\alpha^{*}, \beta^{*}, \gamma^{*}, \delta^{*}, \epsilon^{*}\right)$ represents its optimal dual solution. See Example 1. The reduced cost, $\bar{c}_{S}^{j}$, of column $y_{S}^{j}$, namely $\bar{c}_{S}^{j}=c_{S}^{j}-z_{S}^{j}$ is given by:

$$
\bar{c}_{S}^{j}=c_{S}^{j}+\gamma_{j}^{*}+\delta^{*}+\sum_{i^{\prime} \in I} \sum_{j^{\prime} \in I}\left(\sum_{\substack{(i, k) \in S: \\ r^{\prime} j^{\prime} \geq r_{i j} \\ k \neq 1}} \epsilon_{k}^{*}+\sum_{\substack{(i, k) \in S: \\ r^{\prime} j^{\prime} \leq r_{i j} \\ k \neq n}} \epsilon_{k+1}^{*}\right)-\sum_{\substack{i \in I: \\(i, \cdot) \in S}} \alpha_{i}^{*}-\sum_{\substack{k \in K \\(\cdot, k) \in S}} \beta_{k}^{*} .
$$

If $\bar{c}_{S}^{j} \geq 0$ for all $j, S \in \mathcal{S}(j)$ the current solution of ReLRMP is also optimal for the LRMP and the column generation procedure stops.

Otherwise, one has identified one (some) new column(s) to be added to the current reduced master problem to proceed further. In each iteration, ReLRMP and its reduced costs provide lower and upper bounds for the LRMP. Indeed it holds that (Desrosiers and Lübecke (2005))

$$
\begin{array}{r}
z_{R e L R M P}+p \cdot \min _{j \in I, S \in \mathcal{S}(j)} \bar{c}_{S}^{j} \leq z_{L R M P} \leq z_{R e L R M P} \\
z_{R e L R M P}+\sum_{j \in I} \min _{S \in \mathcal{S}(j)} \bar{c}_{S}^{j} \leq z_{L R M P} \leq z_{\operatorname{ReLRMP}},
\end{array}
$$

where $z_{R e L R M P}$ and $z_{L R M P}$ denote the optimal value of ReLRMP and $L R M P$ respectively.

Example 1. Consider the following vector $\lambda=(4,2,1)$, cost matrix $C$ and precedence matrix $R$ :

$$
C=\left(\begin{array}{lll}
1 & 3 & 6 \\
3 & 1 & 8 \\
6 & 8 & 1
\end{array}\right), \quad R=\left(\begin{array}{lll}
1 & 4 & 6 \\
5 & 2 & 8 \\
7 & 9 & 3
\end{array}\right)
$$


For $n=3$, there are 33 different sets of couples $(i, k)$ in $\mathcal{S}$.

$\begin{array}{llll}S_{1}=\{(1,1)\} & S_{10}=\{(1,1),(2,2)\} & S_{19}=\{(1,3),(2,2)\} & \\ S_{2}=\{(1,2)\} & S_{11}=\{(1,1),(2,3)\} & S_{20}=\{(1,3),(3,1)\} & \\ S_{3}=\{(1,3)\} & S_{12}=\{(1,1),(3,2)\} & S_{21}=\{(1,3),(3,2)\} & S_{28}=\{(1,1),(2,2),(3,3)\} \\ S_{4}=\{(2,1)\} & S_{13}=\{(1,1),(3,3)\} & S_{22}=\{(2,1),(3,2)\} & S_{29}=\{(1,1),(2,3),(3,2)\} \\ S_{5}=\{(2,2)\} & S_{14}=\{(1,2),(2,1)\} & S_{23}=\{(2,1),(3,3)\} & S_{30}=\{(1,2),(2,1),(3,3)\} \\ S_{6}=\{(2,3)\} & S_{15}=\{(1,2),(2,3)\} & S_{24}=\{(2,2),(3,1)\} & S_{31}=\{(1,2),(2,3),(3,1)\} \\ S_{7}=\{(3,1)\} & S_{16}=\{(1,2),(3,1)\} & S_{25}=\{(2,2),(3,3)\} & S_{32}=\{(1,3),(2,1),(3,2)\} \\ S_{8}=\{(3,2)\} & S_{17}=\{(1,2),(3,3)\} & S_{26}=\{(2,3),(3,1)\} & S_{33}=\{(1,3),(2,1),(3,2)\} . \\ S_{9}=\{(3,3)\} & S_{18}=\{(1,3),(2,1)\} & S_{27}=\{(2,3),(3,2)\} & \end{array}$

The sets $\mathcal{S}(j)$ are the following:

$$
\begin{aligned}
& \mathcal{S}(1)=\left\{S_{1}, S_{2}, S_{3}, S_{4}, S_{5}, S_{6}, S_{7}, S_{8}, S_{9}, S_{10}, S_{11}, S_{12}, S_{13}, S_{15}, S_{17}, S_{22}, S_{23}, S_{25}, S_{28}\right\}, \\
& \mathcal{S}(2)=\left\{S_{1}, S_{2}, S_{3}, S_{4}, S_{5}, S_{6}, S_{7}, S_{8}, S_{9}, S_{12}, S_{13}, S_{14}, S_{17}, S_{18}, S_{19}, S_{22}, S_{23}, S_{25}, S_{30}\right\}, \\
& \mathcal{S}(3)=\left\{S_{1}, S_{2}, S_{3}, S_{4}, S_{5}, S_{6}, S_{7}, S_{8}, S_{9}, S_{10}, S_{11}, S_{15}, S_{16}, S_{20}, S_{21}, S_{24}, S_{26}, S_{27}, S_{31}\right\} .
\end{aligned}
$$

We consider as initial pool of columns the variables $y_{18}^{1}$ and $y_{8}^{3}$. With this set of variables, the ReLRMP is

$$
\begin{aligned}
& (\boldsymbol{R e} \boldsymbol{L} \boldsymbol{R} \boldsymbol{M P}) \min \quad+2 y_{5}^{2}+10 y_{13}^{1} \\
& \text { s.t. } \quad+y_{13}^{1} \geq 1 \quad i=1 \\
& +y_{5}^{2} \geq 1 \quad i=2 \\
& +y_{13}^{1} \geq 1 \quad i=3 \\
& +y_{13}^{1} \geq 1 \quad k=1 \\
& +y_{5}^{2} \quad \geq \quad 1 \quad k=2 \\
& +y_{13}^{1} \geq 1 \quad k=3 \\
& -y_{13}^{1} \geq-1 \quad j=1 \\
& -y_{5}^{2} \quad \geq-1 \quad j=2 \\
& \geq-1 \quad j=3 \\
& \begin{array}{rrrrr}
-y_{5}^{2} & -y_{13}^{1} & \geq & -2 & \\
-8 y_{5}^{2} & -y_{13}^{1} & \geq & -9 & k=2 \\
-2 y_{5}^{2} & -3 y_{13}^{1} & \geq & -9 & k=3 \\
& y & \geq 0 &
\end{array}
\end{aligned}
$$

Actually, we are interested in its dual problem:

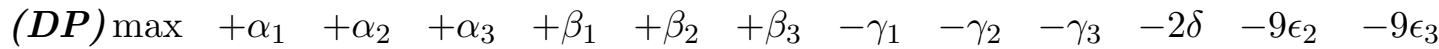

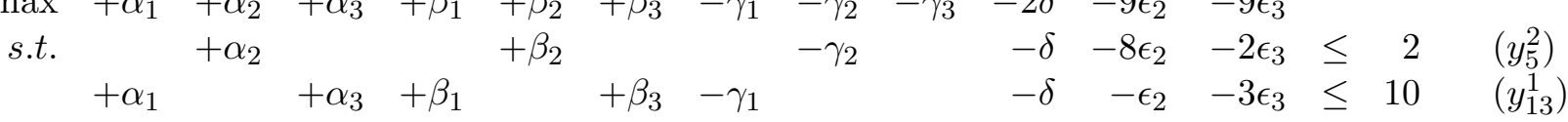

$$
\begin{aligned}
& \alpha, \beta, \quad \gamma, \delta, \epsilon \geq 0 \text {. }
\end{aligned}
$$

Solving (DP), the solution is $\alpha_{2}=2, \beta_{3}=10, \alpha_{1}=\alpha_{3}=\beta_{1}=\beta_{2}=\delta=\epsilon_{2}=\epsilon_{3}=0$ and the value of the objective function is $f=12$.

\subsection{Solving the pricing subproblem}

Although any column $y_{S}^{j}$ with negative reduced cost may be added to ReLRMP, we will follow a strategy that identifies the most negative reduced cost for each facility $j$. This approach 
may give rise to several candidate columns (multiple pricing, see Chvátal (1983)), which is advantageous for this procedure.

In order to do that, for each facility $j \in I$, we solve a subproblem to find the column $y_{S}^{j}$, $S \in \mathcal{S}(j)$, with minimum reduced cost. This set $S$ must be such that there is at most one couple $(i, \cdot)$ for each client $i$ and one $\operatorname{couple}(\cdot, k)$ for each position $k$. Furthermore, the set $S$ must enjoy that the allocation costs of its couples are compatible. We solve this problem by the following dynamic programming algorithm.

Let $d_{i j}^{k}$ be the contribution of the pair $(i, k)$ to the reduced cost of any column $y_{S}^{j}$ such that $(i, k) \in S$. Depending on the values of $k, d_{i j}^{k}$ is given by

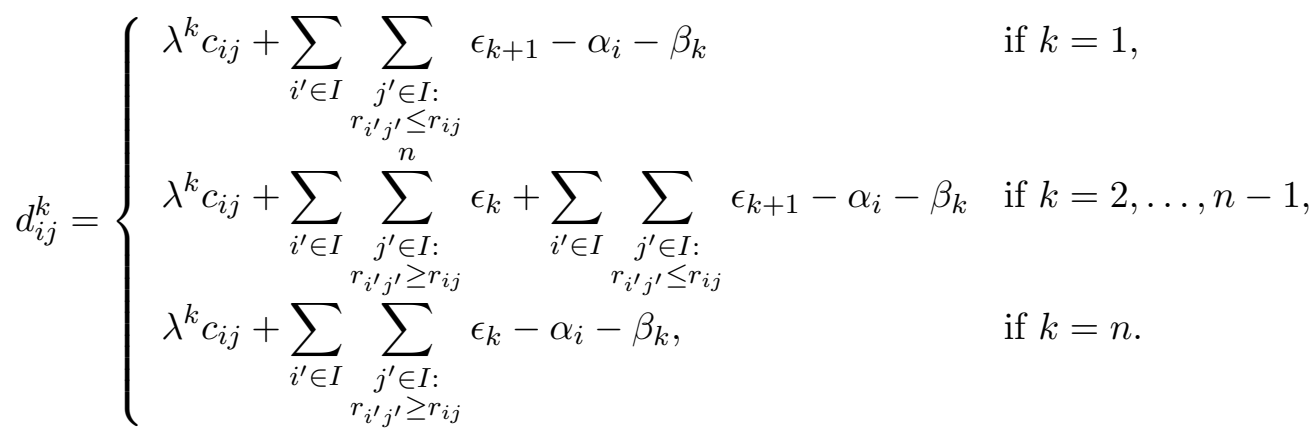

Then, for a facility $j$, the problem of finding the variable $y_{S}^{k}$ with minimum reduced costs can be formulated as:

$$
\min _{S \in \mathcal{S}(j)} \bar{c}_{S}^{j}=\gamma_{j}^{*}+\delta^{*}+\sum_{(i, k) \in S} d_{i j}^{k} .
$$

Now for each facility $j$, we define a matrix $D_{j}$ as follows:

$$
D_{j}=\left(\begin{array}{cccc}
d_{i_{1} j}^{1} & d_{i_{1} j}^{2} & \cdots & d_{i_{1} j}^{n} \\
d_{i_{2} j}^{1} & & & \\
\vdots & & \ddots & \\
d_{i_{n} j}^{1} & & & d_{i_{n} j}^{n}
\end{array}\right)
$$

where $i_{1}, i_{2}, \ldots, i_{n}$ is a permutation of the indices $i=1, \ldots, n$ such that $c_{i_{1} j} \leq c_{i_{2} j} \leq \cdots \leq$ $c_{i_{n} j}$.

Example 2. (Continuing from Example 1). We illustrate the procedure that computes the elements $d_{i j}^{k}$ for all $i, k=1, \ldots, n$ of the matrix $D_{1} \cdot(\boldsymbol{j}=\mathbf{1})$

$$
\begin{array}{ll}
d_{11}^{1}=\lambda^{1} c_{11}+r_{11} \epsilon_{2}-\alpha_{1}-\beta_{1}=4 & d_{11}^{2}=\lambda^{2} c_{11}+\left(n^{2}-r_{11}+1\right) \epsilon_{2}+r_{11} \epsilon_{3}-\alpha_{1}-\beta_{2}=2 \\
d_{11}^{3}=\lambda^{3} c_{11}++\left(n^{2}-r_{11}+1\right) \epsilon_{3}-\alpha_{1}-\beta_{3}=-9 & d_{21}^{1}=\lambda^{1} c_{21}+r_{21} \epsilon_{2}-\alpha_{2}-\beta_{1}=10 \\
d_{21}^{2}=\lambda^{2} c_{21}+\left(n^{2}-r_{21}+1\right) \epsilon_{2}+r_{21} \epsilon_{3}-\alpha_{2}-\beta_{2}=4 & d_{21}^{3}=\lambda^{3} c_{21}++\left(n^{2}-r_{21}+1\right) \epsilon_{3}-\alpha_{2}-\beta_{3}=-9 \\
d_{31}^{1}=\lambda^{1} c_{31}+r_{31} \epsilon_{2}-\alpha_{3}-\beta_{1}=24 & d_{31}^{2}=\lambda^{2} c_{31}+\left(n^{2}-r_{31}+1\right) \epsilon_{2}+r_{21} \epsilon_{3}-\alpha_{3}-\beta_{2}=12 \\
d_{31}^{3}=\lambda^{3} c_{31}++\left(n^{2}-r_{31}+1\right) \epsilon_{3}-\alpha_{3}-\beta_{3}=-4 &
\end{array}
$$

Since $r_{11}<r_{21}<r_{31}$ the valid permutation is $(1,2,3)$. This implies that

$$
D_{1}=\left(\begin{array}{rrr}
4 & 2 & -9 \\
10 & 4 & -9 \\
24 & 12 & -4
\end{array}\right) \begin{aligned}
& i=1 \\
& i=2 \\
& i=3
\end{aligned}
$$


By using $D_{j}$ we obtain that a set $S$ belongs to $\mathcal{S}(j)$ if and only if for every $\left(i_{1}, k_{1}\right)$ and $\left(i_{2}, k_{2}\right) \in S$ such that $i_{1}<i_{2}: k_{1}<k_{2}$.

Our dynamic programming algorithm to obtain the minimum reduced cost for each $j \in J$ builds upon this observation by constructing a solution to a reduced version of (28) in which only the first $i_{l}$ clients and the first $k$ positions are considered.

For each couple $\left(i_{l}, k\right)$, we define a function:

$$
g^{j}\left(i_{l}, k\right)=\min \left\{\bar{c}_{S}^{j}: S \in \mathcal{S}(j) \text { and for all }\left(i_{l}^{\prime}, k^{\prime}\right) \in S: i_{l}^{\prime} \leq i_{l} \text { and } k^{\prime} \leq k\right\}
$$

and we denote an optimal solution of this restricted optimization problem by $S^{j}\left(i_{l}, k\right)$.

Hence, the optimal value of problem (28) is equal to $g^{j}\left(i_{n}, n\right)+\delta+\gamma_{j}$ and a corresponding optimal solution by $S^{j}\left(i_{n}, n\right)$.

Our recursive procedure computes $g^{j}\left(i_{l}, k\right)$ and $S^{j}\left(i_{l}, k\right)$ for increasing values of $l$ and $k$ and exploits the following feasibility conditions on $\mathrm{S}$ :

(i) for each client $i$ (resp. position $k$ ), at most one couple containing $i$ (resp. position $k$ ) belongs to $S$.

(ii) if $\left(i_{l_{1}}, k_{1}\right)$ and $\left(i_{l_{2}}, k_{2}\right) \in S$ and $k_{1}<k_{2}$ then $r_{i_{l_{1}} j}<r_{i_{l_{2}} j}$.

More precisely, if $\left(i_{l}, k\right)$ belongs to $S^{j}\left(i_{l}, k\right)$ then, from (i) it follows that $g^{j}\left(i_{l}, k\right)=$ $g^{j}\left(i_{l-1}, k-1\right)+d_{i_{l} j}^{k}$. Otherwise, $S^{j}\left(i_{l}, k\right)$ may contain a couple $\left(i_{l}, k^{\prime}\right)$ with $k^{\prime} \leq k-1$ or a couple $\left(i_{l^{\prime}}, k\right)$ with $l^{\prime} \leq l-1$ but not both for otherwise condition (ii) would be violated. Hence, in this case, $g^{j}\left(i_{l}, k\right)=\min \left\{g^{j}\left(i_{l-1}, k-1\right), g^{j}\left(i_{l}, k-1\right), g^{j}\left(i_{l-1}, k\right)\right\}$. Combining the two cases, we obtain the following recurrence relation for $l, k=2, \ldots, n$ :

$$
g^{j}\left(i_{l}, k\right)=\min \left\{g^{j}\left(i_{l-1}, k-1\right)+d_{i_{l} j}^{k}, g^{j}\left(i_{l-1}, k-1\right), g^{j}\left(i_{l}, k-1\right), g^{j}\left(i_{l-1}, k\right)\right\} .
$$

Obviously, if at the end of the procedure, $g^{j}\left(i_{n}, n\right)+\delta+\gamma_{j}$ is negative the variable $y_{S^{j}\left(i_{n}, n\right)}^{j}$ is a good candidate to be chosen in the next iteration of the column generation scheme.

If we solve this problem for all $j$, we get $\bar{c}_{R}^{j}=\min _{S} \bar{c}_{S}^{j}$ and if $\bar{c}_{R}^{j}<0$, we can activate (at least) $y_{R}^{j}$. Next, we solve a new reduced master problem ReLRMP with this (these) new activated variable(s).

Remark 1. Computing each matrix $D_{j}$ can be done in $O\left(n^{2}\right)$. Next, obtaining $g^{j}\left(i_{n}, n\right)$ requires the evaluation of the function $g^{j}(i, k)$ for all $i \in I$ and $k \in K$. According to the above algorithm, the evaluation of each $g^{j}(i, k)$ is done in constant time. Solving the pricing subproblem amounts to evaluate $g^{j}\left(i_{n}, n\right)$ for all $j \in I$. Therefore, the entire pricing subproblem can be solved in $O\left(n^{3}\right)$ time.

Example 3. (Continuing from Example 2). We show the computation of the $g^{j}\left(i_{n}, n\right)$ and $S^{j}\left(i_{n}, n\right)$ for $j=1$.

$$
\begin{array}{ll}
g^{1}\left(i_{1}, 1\right)=\min \{0,4\}=0, S^{1}\left(i_{1}, 1\right)=\emptyset & g^{1}\left(i_{1}, 2\right)=\min \{2,0\}=0, S^{1}\left(i_{1}, 2\right)=\emptyset \\
g^{1}\left(i_{1}, 3\right)=\min \{-9,0\}=-9, S^{1}\left(i_{1}, 3\right)=\{(1,3)\} & g^{1}\left(i_{2}, 1\right)=\min \{10,0\}=0, S^{1}\left(i_{2}, 1\right)=\emptyset \\
g^{1}\left(i_{3}, 1\right)=\min \{24,0\}=0, S^{1}\left(i_{3}, 1\right)=\emptyset & g^{1}\left(i_{2}, 2\right)=\min \{0+4,0,0,0\}, S^{1}\left(i_{2}, 2\right)=\emptyset \\
g^{1}\left(i_{3}, 2\right)=\min \{0+12,0,0,0\}, S^{1}\left(i_{3}, 2\right)=\emptyset & g^{1}\left(i_{2}, 3\right)=\min \{0-9,0,-9,0\}, S^{1}\left(i_{2}, 3\right)=\{(1,3)\} \\
g^{1}\left(i_{3}, 3\right)=\min \{0-4,0,-9,0\}, S^{1}\left(i_{3}, 3\right)=\{(1,3)\} &
\end{array}
$$

We have obtained $g^{1}\left(i_{3}, 3\right)=-9$ and $S^{1}\left(i_{3}, 3\right)=S_{3}$ is the potential set to be used, since its reduced cost is negative. The corresponding reduced cost $\bar{c}_{3}^{1}=g^{1}\left(i_{3}, 3\right)+\delta+\gamma_{1}=-9+0+0=$ $-9<0$. Hence, we active variable $y_{3}^{1}$. 


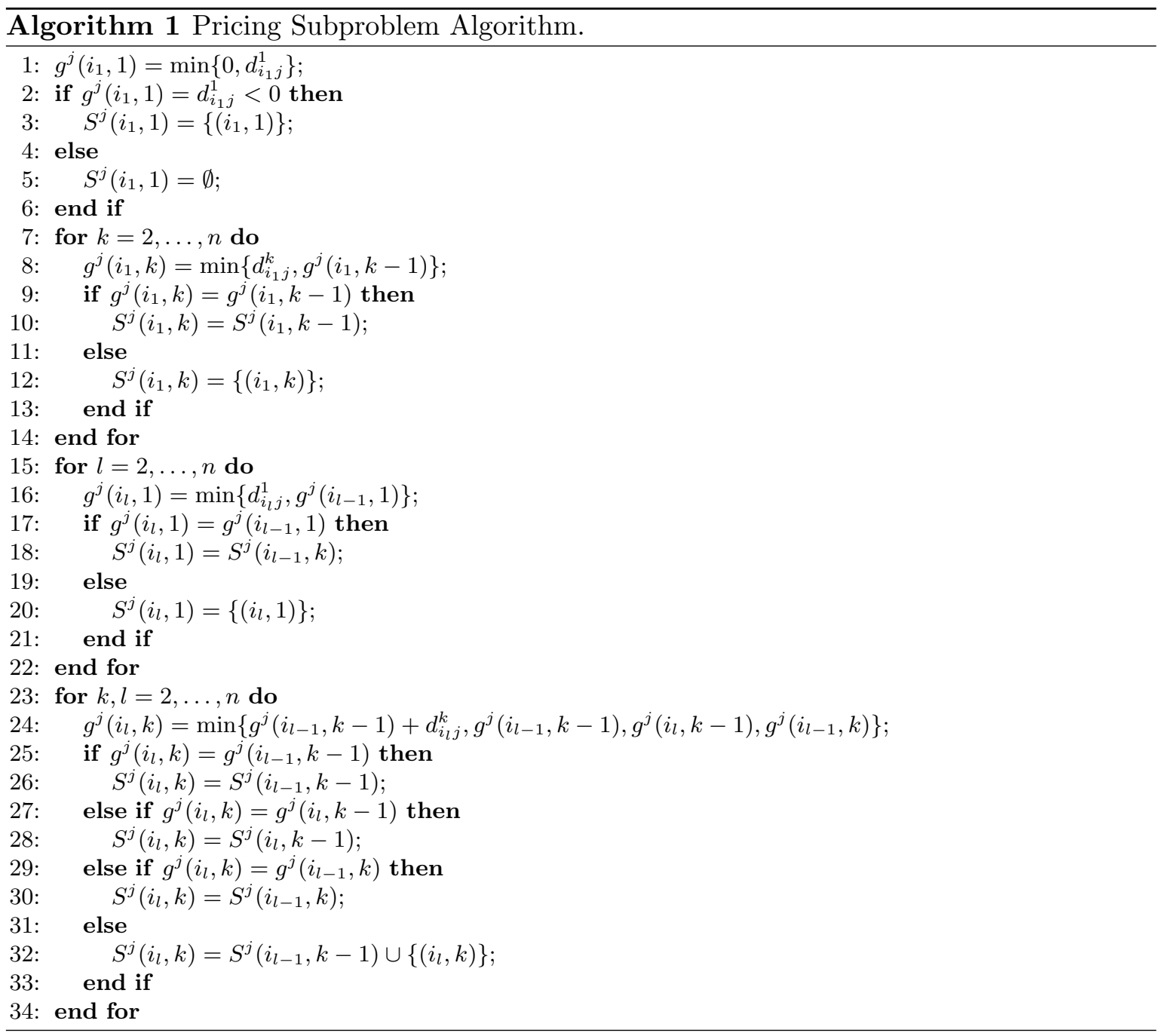

Next, the process continues with the following facilities, i.e. $j=2,3$. In this example the optimal solution can be certified after four complete iterations of the above process.

\subsection{Dealing with infeasibility}

One important issue when implementing a column generation procedure to solve a linear optimization problem is how to deal with infeasibility. This is specially crucial if the procedure is used within a branch-and-bound scheme to solve the linear relaxation of the problem at every node of the branching tree. To handle this, we resort to the so called Farkas pricing. This method was used previously, to the best of our knowledge, in Günlük et al. (2005) and Ceselli et al. (2008). The term Farkas pricing was coined in Gamrath (2010).

According to Farkas' Lemma (Farkas (1894)), a reduced master problem is infeasible if and only if its associated dual problem is unbounded. Thus, to recover feasibility in the ReLRMP we have to revoke the certificate of unboundedness in the dual problem. This can be done by adding constraints to it. Since we are only interested in recovering feasibility in ReLRMP, one can proceed in the same way that for the usual pricing, but with null coefficients in the objective function of the primal. In this way, the Farkas dual problem is

$\max \quad \sum_{i \in I} \alpha_{i}+\sum_{k \in K} \beta_{k}-\sum_{j \in I} \gamma_{j}-p \delta-\sum_{\substack{k \in K: \\ k \neq 1}} n^{2} \epsilon_{k}$ 


$$
\begin{aligned}
& \text { s.t. } \sum_{\substack{i \in I: \\
(i, \cdot) \in S}} \alpha_{i}+\sum_{\substack{k \in K: \\
(\cdot, k) \in S}}^{n} \beta_{k}-\gamma_{j}-\delta-\sum_{i^{\prime} \in I} \sum_{j^{\prime} \in I}\left(\sum_{\substack{i, k) \in S: \\
r^{\prime} j^{\prime} \geq r_{i j} \\
k \neq 1}} \epsilon_{k}+\sum_{\substack{(i, k) \in S: \\
r_{i^{\prime}} j^{\prime} \leq r_{i j} \\
k \neq n}} \epsilon_{k+1}\right) \leq 0 \quad j \in I, S \in \mathcal{S}(j) \\
& \delta, \gamma_{j} \epsilon_{k} \geq 0 \quad j \in I, k \in K, k \neq 1 .
\end{aligned}
$$

To identify new variables that make the reduced master problem feasible we use our dynamic programming approach in which we replace the column $\operatorname{costs} c_{S}^{j}$ by zeros.

Farkas pricing is an important element in our approach because it allows to start the column generation algorithm with an empty pool of columns, although this is not advisable. Furthermore, Farkas pricing will be crucial in the branching phase to recover feasibility (whenever possible) in those nodes of the branching tree where it is lost after fixing variables.

\section{A branch-price-and-cut implementation}

In this section, we precise several components of the implementation of our set partitioning formulation based on a column generation approach. $\mathbf{B} \& \mathbf{P} \& \mathbf{C}$ is a branch-and-cut scheme that solves the linear relaxation at each node of the branching tree with the column generation algorithm previously described and may apply cuts to improve the obtained lower bound. (The reader is referred to Doulabi et al. (2016) for another recent implementation of a $\mathbf{B} \& \mathbf{P} \& \mathbf{C}$.)

Unless otherwise specified, to calibrate the best choice of the different parameters used in our $\mathbf{B} \& \mathbf{P} \& \mathbf{C}$, we have performed a preliminary computational study based on a set of 60 instances with sizes $n=20,30$ and with a time limit of 1800 sec. Those are the smallest instances that we will eventually use in Section 4.

\subsection{Upper bound for the Master Problem: A GRASP heuristic and an initialization stage}

A heuristic algorithm that generates a good feasible solution for $M P$ will provide a promising pool of initial columns as well as a good upper bound.

GRASP (Feo and Resende (1989), Feo and Resende (1995)) is a well-known heuristic technique that usually exhibits good performance in short computing time. In our case, it consists in a multistart greedy algorithm to construct a set of $p$ facilities from a randomly generated set of facilities with smaller cardinality. Following Puerto et al. (2014) we have chosen, in a greedy manner, an initial set of $\lfloor p / 2\rfloor$ facilities. Next, we improve this initial solution by performing a fixed number of iterations of a local search procedure.

The greedy algorithm adds iteratively a new facility to the current set of open facilities, choosing the one with the maximum improvement of the objective value. The local search consists in an interchange heuristic between open and closed facilities. The pseudocode of the GRASP used to solve the problem is described in Algorithm 1. 


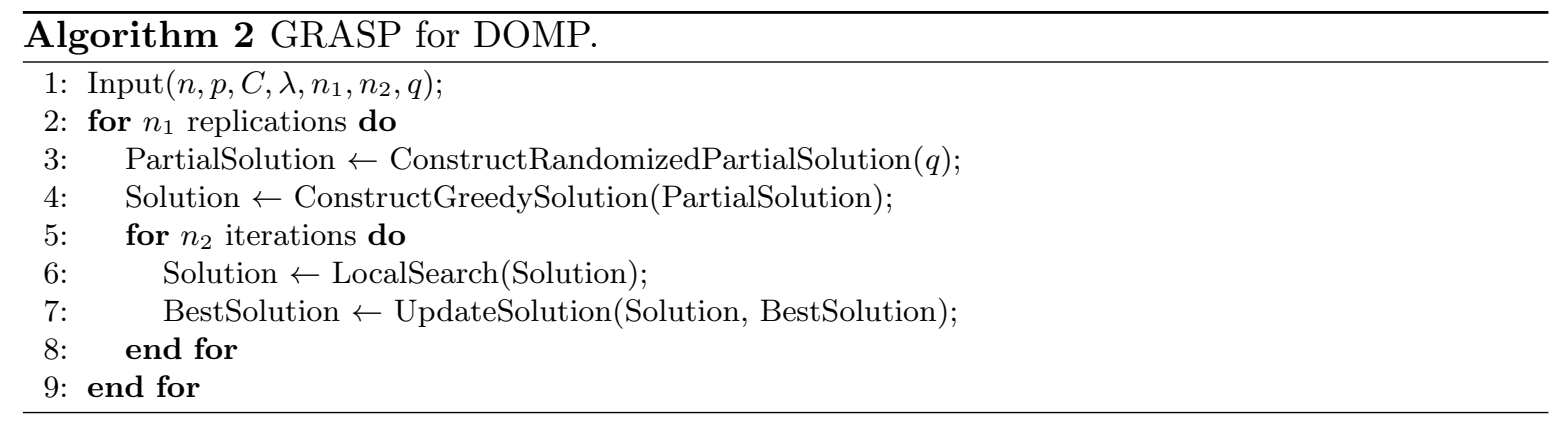

First of all, we would like to point out the remarkable behavior of the GRASP heuristic for this problem. In order to illustrate the appropriateness of our heuristic we have solved to optimality a number of instances of the problem (using the MIP formulation) to be compared with those given by our GRASP. In all instances, up to a size of $n=400$, the solution provided by GRASP is always as good as the one obtained by the any of our MIP formulations with a CPU time limit of 7,200 seconds, see Section 4 .

Moreover, it is not only advisable to use the GRASP heuristic because it provides a very good upper bound thus helping the exploration of the searching tree by pruning many branches of the branch-and-bound tree, but in addition, the construction phase of the heuristic also provides a very promising pool of initial columns for the $\mathbf{B} \& \mathbf{P} \& \mathbf{C}$, in combination with the technique described in the following.

Since we are solving the linear relaxation of our master problem, LRMP, without generating its entire set of variables, using the primal simplex algorithm, the goal of the initialization phase is to find an initial set of columns that allows solving the $M P$ by performing a small number of iterations in the column generation routine. We create variables using a modification of the local search routine of the GRASP algorithm. Every time that we find a promising feasible solution in the heuristic, we create the variables that define that solution (CreateSetVariables $(\mathrm{J})$ ). Algorithm 3 presents the pseudocode of this process.

Function CreateSetVariables $(\mathrm{J})$ determines the costs involved in the solution, i.e. the minimum for each client among the open facilities. Then those costs are ordered to determine the position of each client. Once we know the couples $(i, k)$ assigned for each open facility, the corresponding variables are added to the pool.

Example 4. (Continuing from Example 1) We illustrate the use of the function CreateSetVariables $(J)$ with the following set $J=\{1,3\}$ (open facilities). The allocation costs for this set $J$ of open facilities are $c_{11}=1, c_{21}=3, c_{33}=1$. According to $R$, the ranks of these costs are $r_{11}=1<r_{33}=3<r_{21}=5$. Thus, we get the couples $(1,1),(3,2)$ and $(2,3)$. This means that client 1 goes to facility 1 in position 1, client 3 goes to facility 3 in position 2 and client 2 goes to facility 1 in position 3 . Therefore, the variables $y_{\{(1,1),(2,3)\}}^{1}$ and $y_{\{(3,2)\}}^{3}$ are added to the pool.

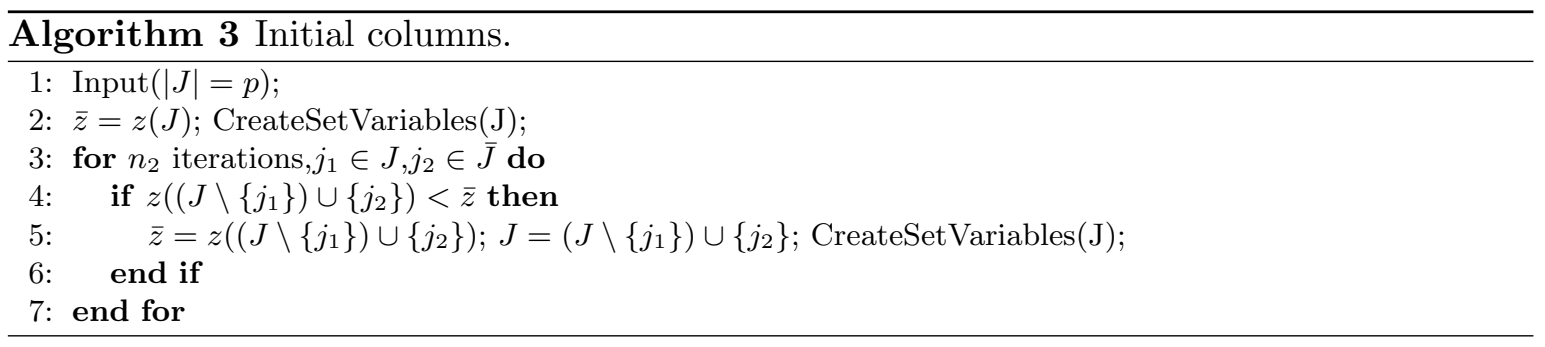

In order to test the usefulness of GRASP in solving problem instances, Table 1 reports 
results for the 60 instances of sizes $n=20,30$ enabling or not the use of the GRASP. It shows average results of CPU time (Time(s)), percent gap at termination, i.e. $100\left(z_{U B}-z_{L B}\right) / z_{L B}$ $(\operatorname{GAP}(\%))$, and number of unsolved problems (in parentheses), number of nodes (\#nodes) and number of variables $(|\operatorname{Vars}|)$.

\begin{tabular}{crrrr}
\hline GRASP & Time(s) & GAP(\%) & \#nodes & $\mid$ Vars $\mid$ \\
\hline Disabled & 1107.21 & $-(36)$ & 158 & 12850 \\
Enabled & 965.31 & $0.89(30)$ & 88 & 9907 \\
\hline
\end{tabular}

Table 1: CPU-Time, Number of nodes and Number of variables with and without GRASP heuristic for $n=20,30$.

\begin{tabular}{cccc}
\hline GRASP & Time(s) & \#nodes & $\mid$ Vars $\mid$ \\
\hline Disabled & 386.19 & 272 & 10962 \\
Enabled & 147.77 & 79 & 6062 \\
\hline
\end{tabular}

Table 2: CPU-Time, Number of nodes and Number of variables with and without GRASP heuristic for $n=20,30$. Summary of solved instances.

According to Table 1 it is clearly advisable to use the upper bound provided by the GRASP heuristic: it reduces the number of nodes, thus improving the size of the branch-and-bound tree.

In Table 2, the same information as in Table 1 is reported but only for the instances solved to optimality within the time limit. One can observe from this table that enabling the use of GRASP reduces the CPU time and number of nodes of the $\mathbf{B} \& \mathbf{B}$ tree and at the same time reduces the overall number of variables required by the $\mathbf{B} \& \mathbf{P} \& \mathbf{C}$. In addition, by using the GRASP heuristic, B\&P\&C is able to solve 6 more instances. For those instances for which $\mathbf{B} \& \mathbf{P} \& \mathbf{C}$ does not certify optimality, GRASP provides an upper bound that leads to an average gap of $0.89 \%$. Finally, without the use of GRASP, in many cases, no feasible solutions are found within the time limit and thus no \% gap ("_") can be reported.

Our results show that by using the GRASP heuristic, $2.03 \%$ of the final number of variables are generated when applying Algorithm 3. The combination of the incumbent solution (given by GRASP) and that initial pool of variables leads to solve the considered instances faster, requiring less number of nodes and variables to certify optimality.

Figure 1 reports the performance profile of GAP versus number of solved instances within a time limit of 1800 seconds, for the 60 instances with sizes $n=20,30$. The blue line reports results using GRASP and the orange one without it. It is interesting to point out that when GRASP is enabled the $\mathbf{B} \& \mathbf{P} \& \mathbf{C}$ is able to solve to optimality 30 instances and the GAP of the remaining never goes beyond $6.43 \%$. On the other hand, if GRASP is disabled then $\mathbf{B} \& \mathbf{P} \& \mathbf{C}$ solves only 24 instances. In addition, it is capable to obtain a feasible solution for only 2 more instances whereas in the remaining 34 instances the gap is greater than $100 \%$ (no feasible solution is found).

\subsection{Stabilization}

When using a column generation procedure, the vector of dual variables may be quite different from an iteration to the next resulting in a slow convergence. For this reason, the stabilization is sometimes a critical step in order to reduce the number of variables and iterations needed to solve each reduced master problem (du Merle et al. (1999)). We follow the stabilization procedure of Pessoa et al. (2010) which depends on only one parameter. The idea consists in using a vector of dual variables which is a convex combination of the previous vector and the current solution of the dual problem.

Let $\pi=(\alpha, \beta, \gamma, \delta, \epsilon)$ be a generic vector of dual multipliers, $\bar{\pi}$ be the best known vector of dual multipliers (found so far) and $\pi_{R e M P}$ be the current solution of the dual problem. Let $\bar{c}_{S}^{j}(\pi)$ be the reduced cost of $y_{S}^{j}$ computed with the dual variable $\pi$ and $L B(\pi)$ the lower 


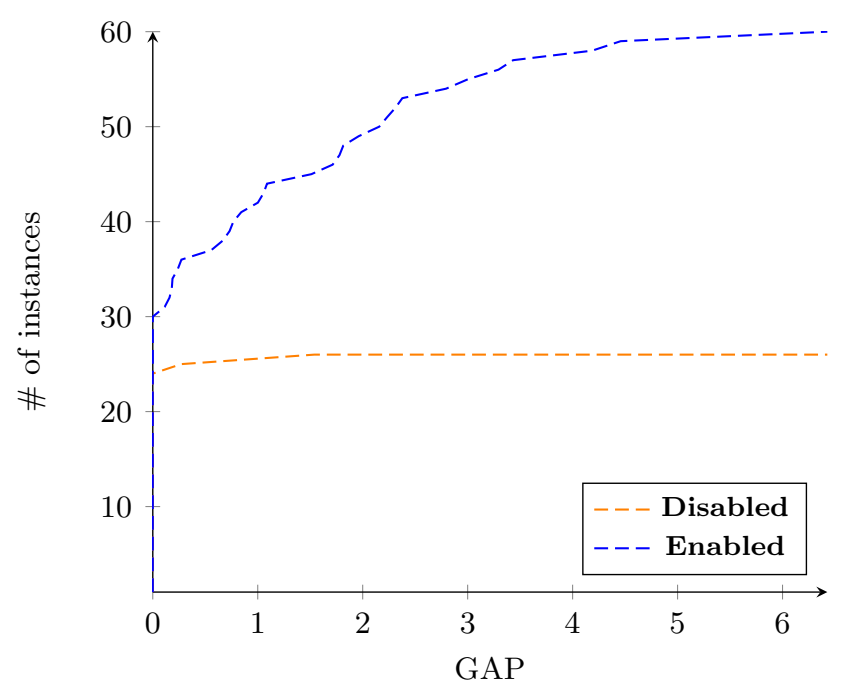

Figure 1: Performance profile graph with GRASP enabled or disabled after 1800 seconds, GAP / \# of instances.

bound provided by the same vector of dual multipliers, namely $\pi$. Finally, let $z_{D}(\pi)$ be the value of the dual objective function of ReLRMP for the dual vector $\pi$, see (25). The stabilization algorithm that we have implemented is described by the following pseudocode:

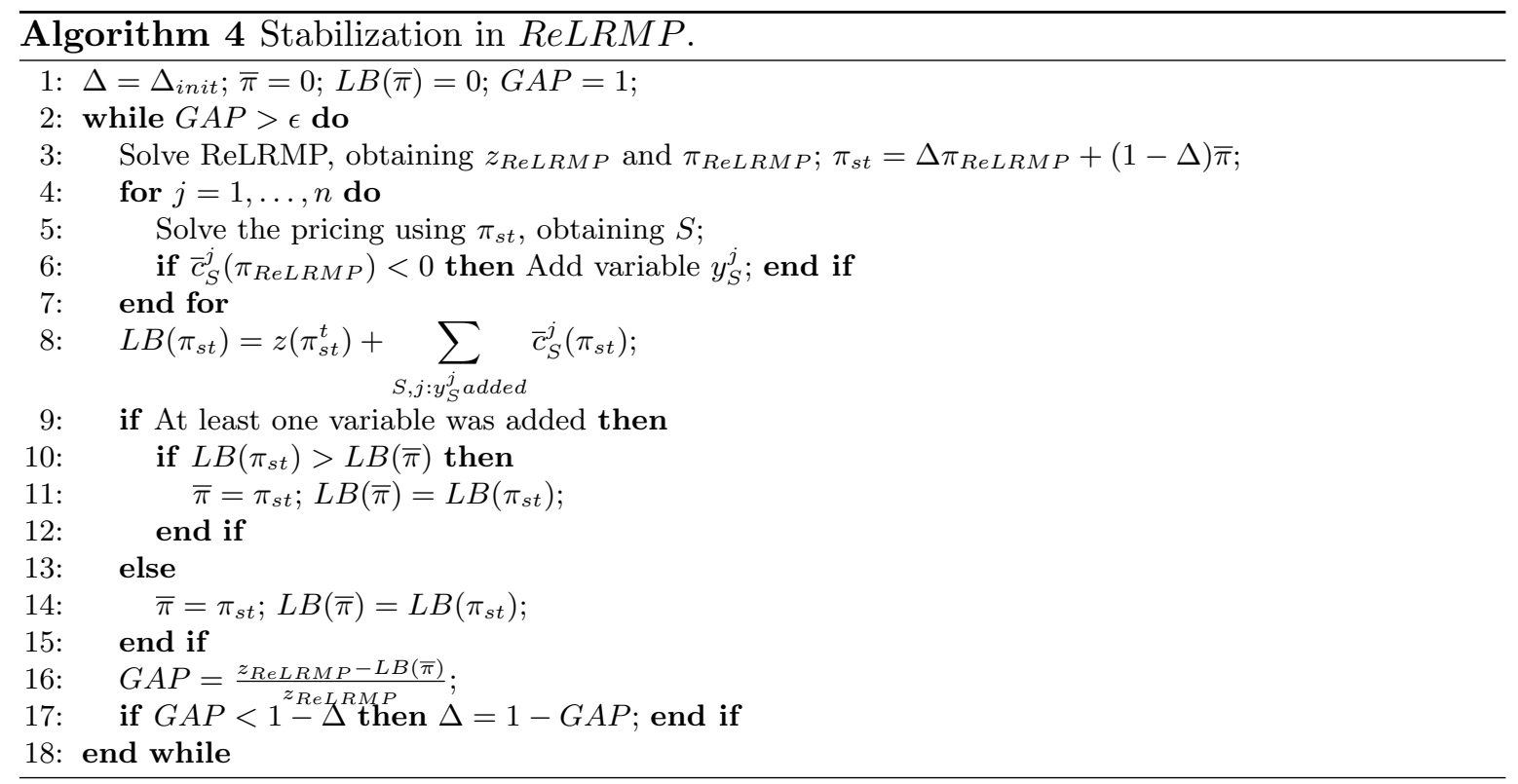

In words, the algorithm performs a while loop where in each iteration it makes a convex combination of the current vector of dual multipliers and the best vector of multipliers found so far. This loop ends whenever both vectors of multipliers are close enough based on the gap between the incumbent lower bound and the actual value of the reduced master problem. It is important to realize that the coefficient (importance), $\Delta$, given in the convex combination to $\pi_{\text {ReLRMP }}$ (the current solution of ReLRMP) increases with the number of iterations of the algorithm since $\Delta=1-G A P$ and $G A P$ decreases with the number of iterations. Eventually in the very last iterations of the stabilization algorithm we will use the actual vector of dual multipliers since $\pi_{s t} \approx \pi_{R e L R M P}$. 
In order to check the efficiency of the stabilization and to determine the best value for parameter $\Delta_{\text {init }}$, LRMP has been solved to optimality for 270 instances from $n=20$ to $n=100$. In our implementation, we have chosen $\Delta=0.4$ based on the computational study shown in Figure 2. As one can observe in this figure, the best performance profile is obtained by $\Delta=0.4$ (blue dashed line) because it is the configuration that solves the instances in less time. It is worth mentioning that LRMP can be solved in one third of the time required for solving the problem without stabilization. Detailed results of the linear relaxation for $\Delta=0.4$ are reported in Table 4 .

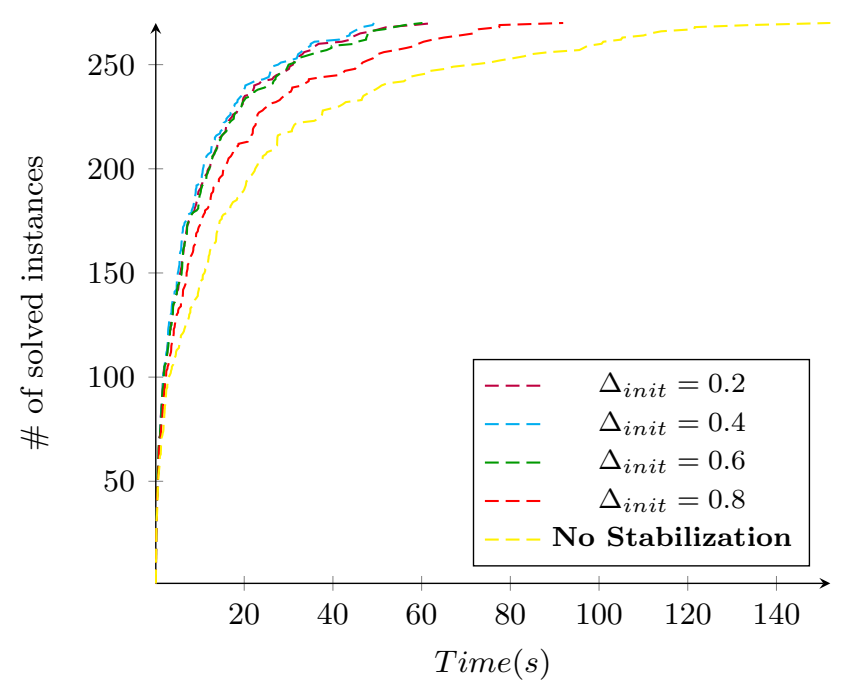

Figure 2: Performance profile graph with different combination of $\Delta_{\text {init }}$, \#solved instances / $n$.

We report in Figure 3 the evolution of the lower and upper bounds with respect to number of iterations for a single instance. Stabilization results in a better behavior: the dual bound is not infinite at iteration 0 and it does not improve for some iterations. The reason is because we start with a feasible solution of the problem.

The control over the dual variables significantly improves the necessary number of iterations and the number of variables used to certify optimality. Note that this improvement becomes more important when $M P$ is solved using a branch-and-bound procedure because

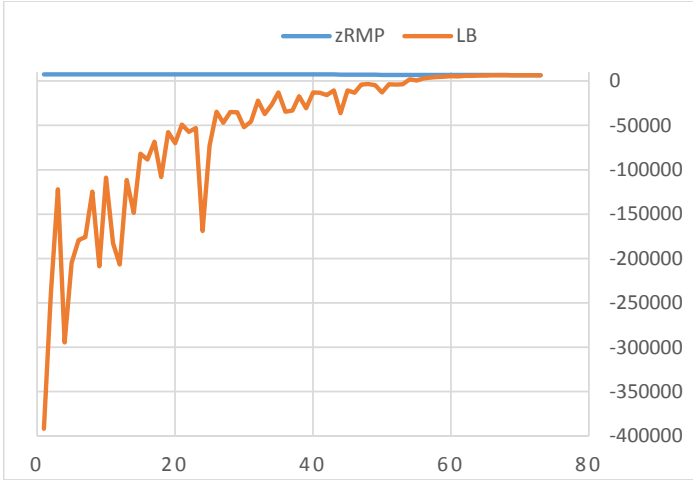

(a) Stabilization disabled

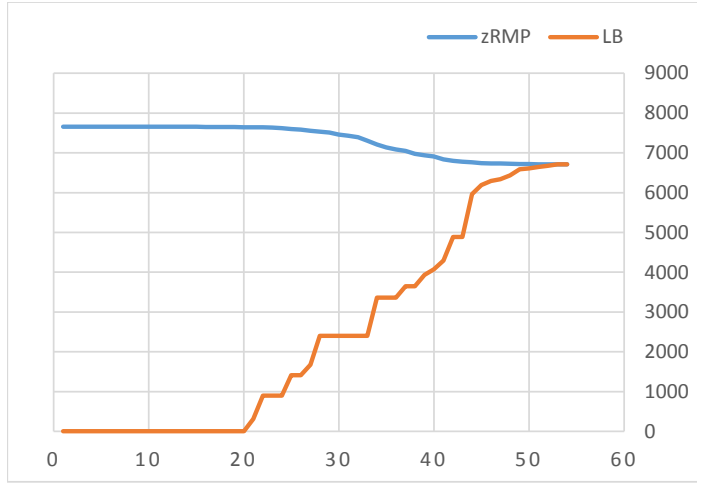

(b) Stabilization enabled

Figure 3: Bound's behavior at the root node in a particular instance on successive iterations. 
the number of variables should be small at every node.

\subsection{Preprocessing}

In order to improve the performance of the algorithm we use two different preprocessings to set some variables to zero. Our approach is based on Claims 1 and 2 in Labbé et al. (2017). The reader may observe that although those results allow to fix some $x_{i j}^{k}$ variables to zero, this variable-fixing can be translated to the new setting by using the relation (10) between the variables in formulations $W O C$ and $M P$.

Therefore, the above results imply that those variables $y_{S}^{j}$ such that $(i, k) \in S$ and $x_{i j}^{k}=0$ will not be considered to be added to the ReLRMP. This can be simply enforced by setting the corresponding $d_{i j}^{k}=0$ in every pricing subproblem.

\subsection{Branching strategies}

Branching on the original variables is a common option when the master problem involves set partition constraints. See for instance Johnson (1989). In spite of that, we have also considered other branching strategies as using the set partitioning variables or the Ryan and Foster branching, Ryan and Foster (1981); Barnhart et al. (1998). However, these two alternatives were discarded because our pricing subproblem is polynomially solvable when we branch on the original variables whereas using any of the other branching strategies mentioned above, makes it $\mathcal{N} \mathcal{P}$-hard.

Recall that $x_{i j}^{k}=\sum_{S \in \mathcal{S}(j):(i, k) \in S} y_{S}^{j}$, thus, a way to branch using a fractional solution can be derived directly from the integrality conditions on the original variables.

Proposition 1. If $x_{i j}^{k} \in\{0,1\}$ for $i, j \in I, k \in K$, then $y_{S}^{j} \in\{0,1\}$ for $j \in I, S \in \mathcal{S}(j)$.

Proof. Suppose on the contrary there exists a variable with fractional value $y_{S^{\prime}}^{j^{\prime}}$. Since $x_{i j}^{k}$ are binary for all $i, j, k$ (in particular for $i_{1}, j^{\prime}, k_{1}$ where $\left(i_{1}, k_{1}\right)$ is a pair of $S^{\prime}$ ), there must be another fractional variable $y_{S^{\prime \prime}}^{j^{\prime}}$ such that $\left(i_{1}, k_{1}\right) \in S^{\prime \prime}$.

Note that $S^{\prime \prime} \neq S^{\prime}$ since the column generation procedure never generates duplicate variables. Hence, there is a pair $\left(i_{2}, k_{2}\right)$ such that either $\left(i_{2}, k_{2}\right) \in S^{\prime}$ or $\left(i_{2}, k_{2}\right) \in S^{\prime \prime}$ but not both. Therefore, we obtain the following relationship

$$
1 \geq \sum_{\substack{S \in \mathcal{S}\left(j^{\prime}\right): \\\left(i_{1}, k_{1}\right) \in S}} y_{S}^{j^{\prime}}>\sum_{\substack{S \in \mathcal{S}\left(j^{\prime}\right): \\\left(i_{2}, k_{2}\right) \in S}} y_{S}^{j^{\prime}}>0 .
$$

The first inequality comes directly from the formulation. The second inequality is strict because the term $\sum_{S \in \mathcal{S}\left(j^{\prime}\right):\left(i_{2}, k_{2}\right) \in S} y_{S}^{j^{\prime}}$ has at least one fractional variable less than the term $\sum_{S \in \mathcal{S}\left(j^{\prime}\right):\left(i_{1}, k_{1}\right) \in S} y_{S}^{j^{\prime}}$. The third inequality is strict because of the choice of $\left(i_{2}, k_{2}\right)$. Finally, a contradiction is found because $x_{i_{2} k_{2}}^{j \prime}$ is not binary.

The reader may note that this branching can be seen as a SOS1 branching (Beale and Tomlin (1970)) since at most one of the above $y_{S}^{j}$ variables can assume the value 1 .

The way to implement this branching in the pricing subproblem is to set locally (in the current node) to zero the $y_{S}^{j}$ variables which are in conflict with the condition implied by the branch $x_{i j}^{k}=0$ or $x_{i j}^{k}=1$.

In the case $x_{i j}^{k}=0$ we set $y_{S}^{j}=0$ for all sets $S$ containing couples $(i, k) \in S$. Analogously, in the case $x_{i j}^{k}=1$ we set $y_{S}^{j^{\prime}}=0$ for all sets $S$ containing $(i, k) \in S$ such that $j \neq j^{\prime}$, $\left(i^{\prime}, k\right) \in S$ such that $i \neq i^{\prime}$ or $\left(i, k^{\prime}\right) \in S$ such that $k \neq k^{\prime}$. 
This condition can be transferred to the pricing subproblem modifying the $d_{i j}^{k}$ coefficients accordingly. Specifically, this transformation is done as follows:

- If $x_{i j}^{k}=0$ then $d_{i j}^{k}=0$.

- If $x_{i j}^{k}=1$ then $\begin{cases}d_{i j^{\prime}}^{k}=0, & j^{\prime} \in I: j^{\prime} \neq j . \\ d_{i^{\prime} j^{\prime}}^{k}=0, & j^{\prime}, i^{\prime} \in I: i^{\prime} \neq i . \\ d_{i j^{\prime}}^{k}=0, & j^{\prime} \in I, k^{\prime} \in K: k^{\prime} \neq k .\end{cases}$

Moreover, it is also well-known that branching on SOS constraints (original variables) gives rise to more balanced branching trees (see e.g. Chapter 7 of Wolsey (1998)) than branching on the variables of $M P$.

Among the fractional original variables one has to decide which will be the next variable to branch on. One of the easiest techniques for this choice is to consider the most fractional variable. This is not difficult to implement but it is not better than choosing randomly (Achterberg et al. (2005)). Alternative techniques are pseudocost branching (Benichou et al. (1971)) or strong branching (Applegate et al. (1995)) although they are rather costly.

This issue has motivated us to propose another rule to select the variable to branch on, based on the improvement of the bounds in each of the new created nodes. We use the following indices corresponding to the down and up branches of the variable $x_{i j}^{k}$ :

$$
\varsigma_{i j}^{k,-}=\frac{\lambda^{k} c_{i j}}{x_{i j}^{k}} \text { and } \varsigma_{i j}^{k,+}=\frac{\lambda^{k} c_{i j}}{1-x_{i j}^{k}} .
$$

They account, respectively, for the unitary contribution to the objective function due to fixing the variable $x_{i j}^{k}$ either to zero (down branching) or to one (up branching). Branching down stimulates the improvement of the lower bound, whereas branching up helps the problem to find integer solutions.

We have tested several strategies that make use of the indices, $\varsigma$, defined above.

Strategy 1: $\arg \min \left\{\theta \varsigma_{i j}^{k,-}+(1-\theta) \varsigma_{i j}^{k,+}: 0<x_{i j}^{k}<1\right\}$.

Strategy 2: $\arg \min \left\{\min \left\{\varsigma_{i j}^{k,-}, \varsigma_{i j}^{k,+}\right\}: 0<x_{i j}^{k}<1\right\}$.

Strategy 3: $\arg \min \left\{\max \left\{\varsigma_{i j}^{k,-}, \varsigma_{i j}^{k,+}\right\}: 0<x_{i j}^{k}<1\right\}$.

Based on our computational experience (see Figure 4), we have concluded that the best strategy to choose the following variable to branch on corresponds to strategy 1 with $\theta=0.5$.

Each node of the branching tree can be fathomed before it is fully processed comparing lower bounds, as given by (25) and (26), with the current incumbent solution. This strategy implies reducing the number of calls to the pricing subproblem and as a result savings in the number of variables added to the restricted master problem.

\subsection{Valid inequalities}

Clearly, the addition of valid inequalities (20) to $M P$ modifies the structure of the master problem and thus the pricing must be modified accordingly. Let us denote by $\zeta_{i j}^{k}$ the dual variable associated with valid inequality (20) for indices $i, j, k$. After some calculation, one 


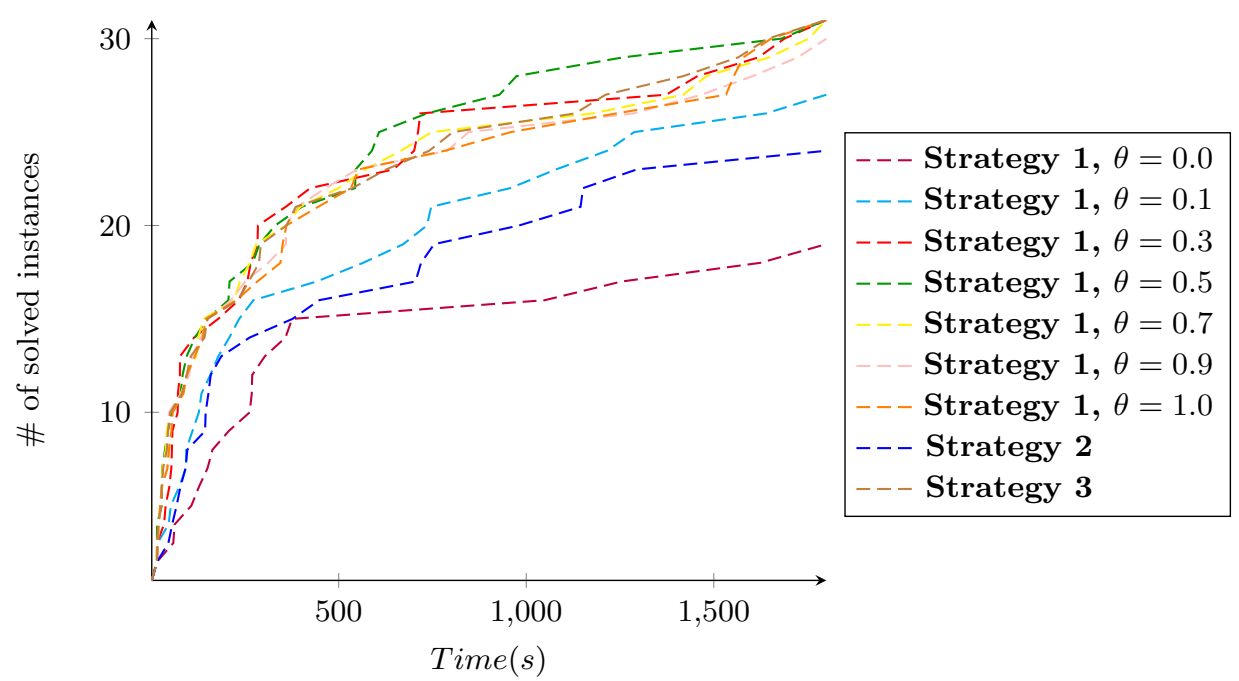

Figure 4: Performance profile graph of \#solved instances using different branching strategies.

obtains the following expression of the reduced costs of variable $y_{S}^{j}$ :

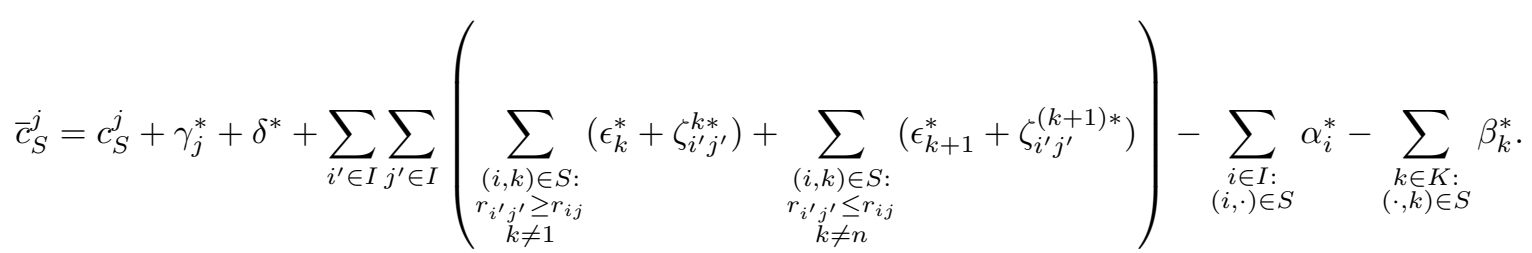

Furthermore, solving the pricing subproblem to find a new column or to certify optimality of the column generation algorithm requires to adapt the dynamic programming algorithm that computes the $g\left(i_{l}, k\right)$ terms using the new dual multipliers. This implies to modify the $D_{j}$ matrices. Once again, after some calculations the modified $d_{i j}^{k}$ elements are now given by:

$d_{i j}^{k}= \begin{cases}\lambda^{k} c_{i j}+\sum_{i^{\prime} \in I} \sum_{\substack{j^{\prime} \in I: \\ \lambda_{i^{\prime} j^{\prime} \leq r_{i j}}}}\left(\epsilon_{k+1}+\zeta_{i^{\prime} j^{\prime}}^{k+1}\right)-\alpha_{i}-\beta_{k} & \text { if } k=1, \\ \lambda^{k} c_{i j}+\sum_{i^{\prime} \in I} \sum_{\substack{j^{\prime} \in I: \\ r_{i^{\prime} j^{\prime} \geq r_{i j}}}}^{k}\left(\epsilon_{k}+\zeta_{i^{\prime} j^{\prime}}^{k}\right)+\sum_{i^{\prime} \in I} \sum_{\substack{j^{\prime} \in I: \\ r_{i^{\prime} j^{\prime}} \leq r_{i j}}}^{k}\left(\epsilon_{k+1}+\zeta_{i^{\prime} j^{\prime}}^{k+1}\right)-\alpha_{i}-\beta_{k} & \text { if } k=2, \ldots, n-1, \\ \lambda^{k} c_{i j}+\sum_{i^{\prime} \in I} \sum_{\substack{j^{\prime} \in I: \\ r_{i^{\prime} j^{\prime}} \geq r_{i j}}}^{\left.k+\epsilon_{k}+\zeta_{i^{\prime} j^{\prime}}\right)-\alpha_{i}-\beta_{k},} & \text { if } k=n .\end{cases}$

These new elements allow us to apply the adapted column generation algorithm to solve LRMP, reinforced with valid inequalities (20).

To justify the use of the mentioned cuts we have done some preliminary computational experiments with instances of sizes $n=50$ and 60 . Table 3 compares the behavior of the standard branch-and-price without cuts, $(\mathbf{B} \& \mathbf{P}(M P))$, against the strategy with cuts, $\mathbf{B} \& \mathbf{P} \& \mathbf{C}(M P)$.

From Table 3, we conclude that it is always better to add cuts because the final gap is always smaller with this strategy. This solution scheme has been implemented and the results are reported in the next section. 


\begin{tabular}{ll|rrr|rrr} 
& & \multicolumn{3}{|c}{$n=50$} & \multicolumn{3}{c}{$n=60$} \\
& & $p=12$ & $p=16$ & $p=25$ & $p=15$ & $p=20$ & $p=30$ \\
\hline B\&P & Time $(s)$ & 7200.03 & 7200.01 & 7200.01 & 7200.07 & 7200.02 & 7202.00 \\
$(\mathrm{MP})$ & $\mid$ Vars $\mid$ & 48648 & 38368 & 21558 & 49990 & 34262 & 24730 \\
& $\mid$ Nodes $\mid$ & 4828 & 11768 & 49523 & 5329 & 12526 & 30305 \\
& \#unsolved & 10 & 10 & 10 & 10 & 10 & 10 \\
& Gap $(\%)$ & 6.26 & 7.57 & 9.57 & 8.29 & 8.97 & 12.07 \\
\hline B\&P\&C & Time $(s)$ & 7200.39 & 7200.49 & 6860.93 & 7200.08 & 7200.77 & 7200.20 \\
$(\mathrm{MP})$ & $\mid$ Vars $\mid$ & 14807 & 14977 & 13407 & 17131 & 16691 & 16838 \\
& $\mid$ Nodes $\mid$ & 1 & 6 & 9 & 2 & 1 & 11 \\
& $\mid$ Cuts $\mid$ & 3526 & 3066 & 2709 & 3489 & 4192 & 2864 \\
& \#unsolved & 10 & 10 & 9 & 10 & 10 & 10 \\
& Gap $(\%)$ & 2.83 & 2.89 & 1.87 & 5.04 & 4.52 & 4.47 \\
\hline
\end{tabular}

Table 3: Numerical results with and without cuts.

\section{Computational Experiments}

The $\mathbf{B} \& \mathbf{P} \& \mathbf{C}$ implementation of the formulation $M P$ has been experimentally compared with the $\mathbf{B} \& \mathbf{C}$ implementation of the formulation $W O C$ on the instances detailed below. The $\mathbf{B} \& \mathbf{P} \& \mathbf{C}$ algorithm considered in these experiments is based on the description in the previous section.

The computer used for these tests has an Intel Core i7 CPU clocked at $2.8 \mathrm{GHz}$ with $4 \mathrm{~GB}$ of RAM. Each implementation has a maximum of 7,200 seconds (2 hours) to solve each individual instance.

Both implementations are using the SCIP 4.0.1's API (see Gamrath et al. (2016)) and both are calling the LP solver of IBM ILOG CPLEX 12.7.

\subsection{Instances}

Since no standard libraries of instances for DOMP are available in public repositories we generate our own instances with the pseudorandom number generator from the $\mathrm{C}$ random library. In this work we consider that the sets of clients and potential facilities coincide, thereby we will refer both as points.

We consider 20 sets of 30 instances. Each set has a different number of points such that $n \in\{20,30, \ldots, 90,100,120,140, \ldots, 280,300,400\}$. For a given $n$, we generate one subset of 10 instances for each value of $p$, where $p \in\{\lfloor(n / 4)\rfloor,\lfloor(n / 3)\rfloor,\lfloor(n / 2)\rfloor\}$.

For a given $n$, we first randomly generate the Cartesian coordinates of the points in the square $[0,400]^{2}$. Then, we calculate the cost for each pair of points with the Euclidean distance between the two related nodes in the square. We round each distance to the nearest integer to build the cost matrices. We also fix the values of the matrix diagonal to the smallest admissible cost to avoid free self service.

Finally, we randomly generate the weight vector $\lambda$ such that $\lambda^{k} \in[n / 4, n]$ for $k \in$ $K$. All these instances, with $n$ up to 400, are available at https://gom.ulb.ac.be/gom/ wp-content/uploads/2018/12/DOMP_Repository.zip. Detailed information about the instances generation can be found in Deleplanque et al. (2018).

\section{2 $\quad M P$ vs $W O C$ linear relaxations}

We assess experimentally the linear relaxation of $M P$ by comparing with $W O C$ on all the instances generated. For these experiments, neither cuts nor preprocessing have been applied. 
In Table 4, we report averages of the numerical results of the linear relaxation for both formulations. The value $\operatorname{Gap} L P(\%)$ represents the gap percentage between the optimal integer value $z^{*}$ (alternatively the best known solution) and the linear relaxation optimal value $z_{L P}^{*}: \operatorname{Gap} L P(\%)=100\left(z^{*}-z_{L P}^{*}\right) / z_{L P}^{*}$. The computational times in the column Time $(s)$ are given in seconds. Table 4 also includes average number of variables $(\mid$ Vars $\mid)$ and required memory $(\operatorname{Memory}(M B))$. We highlight the small number of variables that are generated to certify optimality with the column generation approach applied to $M P$, besides the time and memory saving, which is likewise significant.

\begin{tabular}{|c|c|c|c|c|c|c|c|c|c|}
\hline \multirow[b]{2}{*}{$n$} & \multirow[b]{2}{*}{$p$} & \multicolumn{4}{|c|}{$($ WOC $)$} & \multicolumn{4}{|c|}{$(\mathrm{MP})$} \\
\hline & & $\operatorname{Gap} L P(\%)$ & Time $(s)$ & $\mid$ Vars $\mid$ & Memory $(M B)$ & $\operatorname{Gap} L P(\%)$ & Time $(s)$ & $\mid$ Vars $\mid$ & Memory $(M B)$ \\
\hline 20 & 10 & 15.35 & 0.12 & 8020 & 35 & 14.78 & 0.09 & 450 & 2 \\
\hline 30 & 15 & 16.77 & 0.62 & 27030 & 101 & 16.16 & 0.29 & 1067 & 5 \\
\hline 40 & 20 & 18.22 & 2.09 & 64040 & 235 & 17.98 & 0.74 & 2005 & 10 \\
\hline 50 & 25 & 15.02 & 6.25 & 125050 & 451 & 14.74 & 1.36 & 2922 & 15 \\
\hline 60 & 30 & 16.94 & 12.27 & 216060 & 764 & 16.69 & 2.74 & 4777 & 23 \\
\hline 70 & 35 & 15.97 & 29.40 & 343070 & 1214 & 15.80 & 4.61 & 6327 & 30 \\
\hline 80 & 40 & 7.67 & 47.63 & 512080 & 1830 & 7.63 & 5.63 & 7161 & 34 \\
\hline 90 & 45 & 7.19 & 82.19 & 729090 & 2561 & 7.15 & 9.14 & 8867 & 42 \\
\hline 100 & 50 & - & - & 1000100 & $>4096$ & 7.05 & 14.18 & 11255 & 54 \\
\hline 20 & 6 & 9.56 & 0.14 & 8020 & 35 & 8.79 & 0.14 & 548 & 3 \\
\hline 30 & 10 & 11.68 & 0.68 & 27030 & 101 & 10.96 & 0.39 & 1257 & 7 \\
\hline 40 & 13 & 12.39 & 2.34 & 64040 & 235 & 12.08 & 1.17 & 2371 & 14 \\
\hline 50 & 16 & 9.65 & 6.37 & 125050 & 451 & 9.34 & 2.09 & 3641 & 21 \\
\hline 60 & 20 & 11.11 & 13.30 & 216060 & 764 & 10.82 & 4.18 & 5652 & 32 \\
\hline 70 & 23 & 10.16 & 35.65 & 343070 & 1214 & 9.98 & 7.54 & 7684 & 44 \\
\hline 80 & 26 & 8.32 & 58.14 & 512080 & 1830 & 8.11 & 11.32 & 9699 & 55 \\
\hline 90 & 30 & 7.08 & 96.74 & 729090 & 2561 & 7.02 & 17.58 & 12185 & 69 \\
\hline 100 & 33 & - & - & 1000100 & $>4096$ & 8.01 & 28.76 & 15656 & 87 \\
\hline 20 & 5 & 9.53 & 0.14 & 8020 & 35 & 8.61 & 0.16 & 574 & 4 \\
\hline 30 & 7 & 10.61 & 0.70 & 27030 & 101 & 9.36 & 0.59 & 1278 & 9 \\
\hline 40 & 10 & 10.44 & 2.51 & 64040 & 235 & 10.05 & 1.61 & 2536 & 17 \\
\hline 50 & 12 & 7.96 & 7.35 & 125050 & 451 & 7.54 & 3.01 & 3955 & 27 \\
\hline 60 & 15 & 9.74 & 15.98 & 216060 & 764 & 9.41 & 5.56 & 5697 & 36 \\
\hline 70 & 17 & 8.80 & 40.78 & 343070 & 1214 & 8.50 & 10.19 & 7828 & 53 \\
\hline 80 & 20 & 9.53 & 67.42 & 512080 & 1830 & 9.38 & 17.38 & 10740 & 69 \\
\hline 90 & 22 & 9.58 & 128.70 & 729090 & 2561 & 9.41 & 28.66 & 13581 & 86 \\
\hline 100 & 25 & - & - & 1000100 & $>4096$ & 8.65 & 44.20 & 17084 & 106 \\
\hline
\end{tabular}

Table 4: Numerical results on linear relaxation for $W O C$ and $M P$.

As expected, the integrality gap of formulation $M P$ is smaller than the one of $W O C$. Moreover, formulation $M P$ also outperforms $W O C$ in the number of required variables (see Figure 5) which results in much smaller memory requirements (see Figure 6). Indeed, the implementation of $W O C$ fails to solve, already for sizes of $n=100$, the linear relaxation of all instances by lack of RAM memory; whereas with the same parameter configuration, formulation $M P$ is relatively far from experiencing that problem. Figure 6 shows the performance profile of the memory requirement of both formulations. As one can see $M P$ outperforms $W O C$ with respect to this factor for all instance sizes.

\section{$4.3 \quad \mathrm{~B} \& \mathrm{P} \& \mathrm{C}(\mathrm{MP})$ vs $\mathrm{B} \& \mathrm{C}(\mathrm{WOC})$}

We now compare the $\mathbf{B} \& \mathbf{P} \& \mathbf{C}$ implementation of $M P$ with the $\mathbf{B} \& \mathbf{C}$ implementation of $W O C$. The former follows the procedure explained in Section 3 and the latter consists of $W O C$ formulation with (9) as valid inequalities.

The results are reported in Table 5. In that table, we denote by Time $(s)$ the average computational time (in seconds) required by each method to obtain an optimal solution for a given set of 10 instances defined by number of clients $(n)$ and number of open facilities $(p)$. 

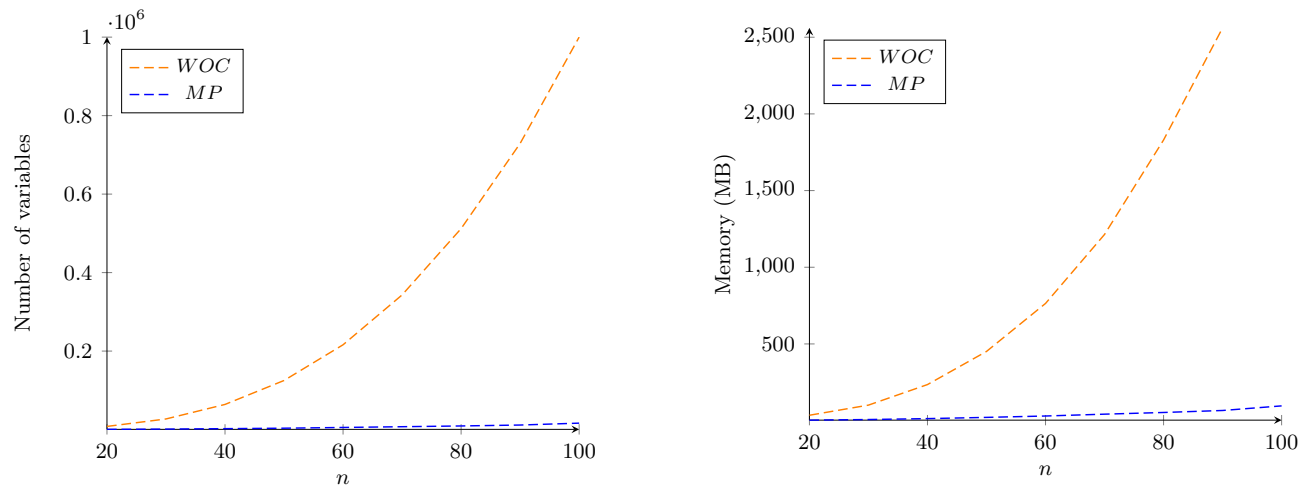

Figure 5: Graph of Number of Variables ver- Figure 6: Graph of Memory usage (MB versus size $n$ for $W O C$ and $M P$. sus size $n$ for $W O C$ and $M P$.

\begin{tabular}{|c|c|c|c|c|c|c|c|c|c|c|c|c|c|}
\hline \multirow[b]{2}{*}{$n$} & \multirow[b]{2}{*}{$p$} & \multicolumn{6}{|c|}{$\mathbf{B} \& \mathbf{C}($ WOC $)$} & \multicolumn{6}{|c|}{$\mathbf{B} \& \mathbf{P} \& \mathbf{C}(\mathrm{MP})$} \\
\hline & & Time $(s)$ & $\mid$ Vars $\mid$ & $\mid$ Nodes $\mid$ & $\mid$ Cuts $\mid$ & \#unsolved $(T / M)$ & $\operatorname{Gap}(\%)$ & Time $(s)$ & $\mid$ Vars $\mid$ & $\mid$ Nodes $\mid$ & $\mid$ Cuts $\mid$ & \#unsolved & $\operatorname{Gap}(\%)$ \\
\hline 20 & 10 & 4.48 & 4211 & 38 & 689 & $0 / 0$ & 0.00 & 58.70 & 4272 & 111 & 373 & 0 & 0.00 \\
\hline 30 & 15 & 131.89 & 13952 & 19197 & 2519 & $0 / 0$ & 0.00 & 3670.21 & 16369 & 392 & 1078 & 3 & 0.45 \\
\hline 40 & 20 & 6202.85 & 32820 & 605812 & 4727 & $8 / 0$ & 2.54 & 6751.27 & 14591 & 41 & 1819 & 9 & 3.33 \\
\hline 50 & 25 & 6575.59 & 63776 & 355560 & 10131 & $9 / 0$ & 1.35 & 6860.93 & 13407 & 9 & 2709 & 9 & 1.87 \\
\hline 60 & 30 & 6707.38 & 109804 & 85723 & 15676 & $8 / 2$ & 1.82 & 7200.20 & 16838 & 11 & 2864 & 10 & 4.47 \\
\hline 70 & 35 & 2474.98 & 173955 & 835 & 19238 & $2 / 8$ & 7.72 & 7200.31 & 17758 & 2 & 4150 & 10 & 4.95 \\
\hline 80 & 40 & 3428.13 & 259186 & 1 & 12406 & $0 / 10$ & 3.38 & 7201.36 & 18902 & 2 & 4680 & 10 & 2.13 \\
\hline 90 & 45 & 6243.49 & 368560 & 1 & 12157 & $7 / 3$ & 4.24 & 7200.38 & 20028 & 7 & 4127 & 10 & 2.37 \\
\hline 20 & 6 & 11.50 & 5706 & 440 & 1249 & $0 / 0$ & 0.00 & 952.76 & 10959 & 97 & 615 & 0 & 0.00 \\
\hline 30 & 10 & 1578.21 & 18245 & 305595 & 3056 & $1 / 0$ & 0.12 & 6270.41 & 17502 & 81 & 1503 & 8 & 1.61 \\
\hline 40 & 13 & 7061.36 & 43664 & 628962 & 6559 & $8 / 2$ & 2.38 & 7200.72 & 11186 & 2 & 3073 & 10 & 3.69 \\
\hline 50 & 16 & 7116.54 & 85630 & 284028 & 10423 & $9 / 1$ & 1.14 & 7200.49 & 14977 & 6 & 3066 & 10 & 2.89 \\
\hline 60 & 20 & 3306.54 & 144983 & 20330 & 19887 & $2 / 8$ & 3.02 & 7200.77 & 16691 & 1 & 4192 & 10 & 4.52 \\
\hline 70 & 23 & 2119.13 & 231680 & 1 & 23603 & $0 / 10$ & 6.20 & 7200.97 & 19307 & 2 & 4365 & 10 & 4.51 \\
\hline 80 & 26 & 2886.25 & 346926 & 1 & 25187 & $0 / 10$ & 5.59 & 7201.35 & 21675 & 1 & 5449 & 10 & 3.69 \\
\hline 90 & 30 & 5214.89 & 488316 & 1 & 32406 & $0 / 10$ & 4.62 & 7201.66 & 24507 & 1 & 6116 & 10 & 3.17 \\
\hline 20 & 5 & 16.54 & 6054 & 1215 & 1537 & $0 / 0$ & 0.00 & 1989.43 & 14699 & 97 & 731 & 1 & 0.09 \\
\hline 30 & 7 & 1807.41 & 20643 & 198424 & 4789 & $1 / 1$ & 0.65 & 6840.10 & 14934 & 29 & 2093 & 8 & 1.85 \\
\hline 40 & 10 & 7050.93 & 48065 & 602685 & 7939 & $7 / 3$ & 1.68 & 7200.90 & 10730 & 1 & 3455 & 10 & 3.95 \\
\hline 50 & 12 & 7200.00 & 94784 & 270959 & 12579 & $10 / 0$ & 0.91 & 7201.39 & 14807 & 1 & 3526 & 10 & 2.83 \\
\hline 60 & 15 & 2768.88 & 161807 & 1 & 18081 & $0 / 8$ & 2.90 & 7201.08 & 17131 & 2 & 3489 & 10 & 5.04 \\
\hline 70 & 17 & 1842.00 & 259406 & 1 & 16115 & $0 / 10$ & 6.04 & 7201.78 & 19454 & 1 & 4649 & 10 & 4.51 \\
\hline 80 & 20 & 2902.00 & 383199 & 1 & 27129 & $0 / 10$ & 6.95 & 7201.52 & 25278 & 3 & 4320 & 10 & 4.89 \\
\hline 90 & 22 & 5999.16 & 549561 & 1 & 46216 & $0 / 10$ & 6.82 & 7201.75 & 27418 & 1 & 5549 & 10 & 5.50 \\
\hline
\end{tabular}

Table 5: Numerical results for $\mathbf{B} \& \mathbf{C}(\mathrm{WOC})$ and $\mathbf{B} \& \mathbf{P} \& \mathbf{C}(\mathrm{MP})$.

With $\mid$ Vars $\mid$ we refer to the average of the numbers of variables used by $M P$ or $W O C$. We also denote by $\mid$ Nodes $\mid$ and $\mid$ Cuts $\mid$ the average of the number of nodes explored and the average of the number of cuts used, respectively, in the corresponding methodology. The column \#unsolved $(T / M)$ in the case of $\mathbf{B} \& \mathbf{C}($ WOC) reports the number of unsolved instances out of the 10 in each group. It distinguishes between those instances not solved by exceeding the maximum running time $(T)$ or the memory limits $(M)$. Observe that in the similar column within the blocks $\mathbf{B} \& \mathbf{P} \& \mathbf{C}(\mathrm{MP})$ no distinction is shown since the memory limit is never reached and instances may be not solved only because of time limitation. Finally, we also include the gap at termination as $\operatorname{Gap}(\%)=100\left(z_{U B}-z_{L B}\right) / z_{L B}$, where $z_{L B}$ and $z_{U B}$ are the lower and upper bound, respectively.

Analyzing further the results in Table 5 we conclude that on average $\mathbf{B} \& \mathbf{C}($ WOC) uses less variables than $\mathbf{B} \& \mathbf{P} \& \mathbf{C}(\mathrm{MP})$. This allows us to solve larger sized instances that were not affordable for the original WOC. We also observe that the number of required cuts for $\mathbf{B} \& \mathbf{P} \& \mathbf{C}(\mathrm{MP})$ is smaller than for $\mathbf{B} \& \mathbf{C}($ WOC $)$. This could be explained by the tightness 
of $\mathbf{B} \& \mathbf{P} \& \mathbf{C}(\mathrm{MP})$ with respect to $\mathbf{B} \& \mathbf{C}($ WOC). After adding cuts $\mathbf{B} \& \mathbf{P} \& \mathbf{C}(\mathrm{MP})$ is able to solve the problem with a smaller branch-and-bound tree. The number of instances solved to optimality, for small size instances up to $n=40$, is slightly better for $\mathbf{B} \& \mathbf{C}($ WOC). As the size increases this number is similar in both cases. Gaps at termination, after 7200 seconds, are always smaller than $8 \%$ for $\mathbf{B} \& \mathbf{C}(\mathrm{WOC})$ and smaller than $6 \%$ for $\mathbf{B} \& \mathbf{P} \& \mathbf{C}(\mathrm{MP})$, being the later clearly better from instances of $n=70$. Since $\mathbf{B} \& \mathbf{C}(\mathrm{WOC})$ is not able to handle any instance with $n=100$ (reporting out of memory flags) we continue our study in Table 6 without this formulation.

\begin{tabular}{|c|c|c|c|c|c|c|c|}
\hline$n$ & $p$ & Time $(s)$ & $\mid$ Vars $\mid$ & $\mid$ Nodes $\mid$ & $\mid$ Cuts $\mid$ & Memory $(M B)$ & $\operatorname{Gap}(\%)$ \\
\hline 100 & 50 & 7201.10 & 23057 & 2 & 5221 & 311 & 2.43 \\
\hline 120 & 60 & 7200.54 & 29209 & 1 & 6992 & 424 & 2.54 \\
\hline 140 & 70 & 7202.11 & 34222 & 2 & 6805 & 449 & 2.87 \\
\hline 160 & 80 & 7201.08 & 41811 & 1 & 8069 & 574 & 3.13 \\
\hline 180 & 90 & 7201.24 & 50523 & 1 & 7961 & 656 & 3.48 \\
\hline 200 & 100 & 7201.61 & 59931 & 1 & 9050 & 805 & 3.96 \\
\hline 220 & 110 & 7201.39 & 57685 & 1 & 11097 & 806 & 4.39 \\
\hline 240 & 120 & 7200.94 & 63710 & 1 & 11487 & 874 & 4.43 \\
\hline 260 & 130 & 7201.83 & 73143 & 1 & 9772 & 910 & 5.05 \\
\hline 280 & 140 & 7200.98 & 83892 & 1 & 9592 & 1037 & 6.19 \\
\hline 300 & 150 & 7201.74 & 87444 & 1 & 10811 & 1076 & 6.83 \\
\hline 100 & 33 & 7201.10 & 29974 & 2 & 5864 & 562 & 3.81 \\
\hline 120 & 40 & 7201.12 & 37204 & 5 & 5433 & 621 & 4.80 \\
\hline 140 & 46 & 7200.98 & 48286 & 2 & 7953 & 894 & 4.47 \\
\hline 160 & 53 & 7201.55 & 57060 & 3 & 7159 & 926 & 5.01 \\
\hline 180 & 60 & 7203.85 & 70077 & 1 & 8997 & 1188 & 5.99 \\
\hline 200 & 66 & 7200.55 & 67417 & 1 & 11416 & 1199 & 6.16 \\
\hline 220 & 73 & 7201.41 & 71731 & 2 & 9356 & 1073 & 6.88 \\
\hline 240 & 80 & 7202.02 & 84466 & 2 & 10093 & 1324 & 9.80 \\
\hline 260 & 86 & 7200.74 & 92401 & 1 & 10505 & 1390 & 9.54 \\
\hline 280 & 93 & 7201.68 & 105535 & 1 & 13265 & 1728 & 9.01 \\
\hline 300 & 100 & 7200.28 & 114427 & 1 & 15595 & 2034 & 9.41 \\
\hline 100 & 25 & 7202.69 & 31218 & 1 & 7034 & 801 & 4.73 \\
\hline 120 & 30 & 7200.98 & 40074 & 1 & 6818 & 922 & 5.91 \\
\hline 140 & 35 & 7203.38 & 49539 & 1 & 8466 & 1162 & 5.83 \\
\hline 160 & 40 & 7201.35 & 58464 & 1 & 10688 & 1465 & 7.29 \\
\hline 180 & 45 & 7203.43 & 72146 & 1 & 10329 & 1674 & 8.70 \\
\hline 200 & 50 & 7201.88 & 62568 & 1 & 10252 & 1364 & 11.27 \\
\hline 220 & 55 & 7203.56 & 74359 & 1 & 9985 & 1515 & 10.34 \\
\hline 240 & 60 & 7200.19 & 80228 & 1 & 9745 & 1422 & 11.57 \\
\hline 260 & 65 & 7200.54 & 99628 & 1 & 7944 & 1346 & 11.77 \\
\hline 280 & 70 & 7203.63 & 109544 & 1 & 4187 & 1247 & 11.32 \\
\hline 300 & 75 & 7200.79 & 128462 & 1 & 3844 & 1261 & 12.26 \\
\hline 400 & 200 & ${ }^{1} 86400.42$ & 158287 & $\overline{1}$ & 9308 & 1183 & 7.07 \\
\hline 400 & 133 & 86401.23 & 178265 & 1 & 13652 & 2764 & 11.26 \\
\hline 400 & 100 & 86401.11 & 236973 & 1 & 7582 & 3125 & 10.29 \\
\hline
\end{tabular}

Table 6: Numerical results for $\mathbf{B} \& \mathbf{P} \& \mathbf{C}(\mathrm{MP})$ for bigger instances.

Table 6 contains the results within the time limit of 2 hours for bigger instances of DOMP. This table has the same layout as Table 5 except that we replace column \#unsolved by Memory $(M B)$. This new column shows the average required memory to solve the corresponding set of instances. In that table extensive computational experiments are reported for instances up to 400 points. We would like to remark that the increase of the complexity, with respect to the instance sizes, of $|\operatorname{Vars}|,|\mathrm{Cuts}|, \operatorname{Memory}(M B)$ and $\operatorname{Gap}(\%)$ is moderate (almost linear) which allows one to handle DOMP problems of larger size. Moreover the Gap $(\%)$ are similar to those reported in Table 5.

To conclude, the results show that the overall performance of $\mathbf{B} \& \mathbf{P} \& \mathbf{C}(\mathrm{MP})$ in solving DOMP is systematically better than the branch-and-cut formulation $\mathbf{B} \& \mathbf{C}($ WOC) for instances for $n \geq 70$. In addition, it is worth noting that $\mathbf{B} \& \mathbf{C}$ (WOC) is not even able to solve 
the linear relaxation of DOMP problems of sizes $n \geq 100$. This fact shows the usefulness of our new approach.

\section{Conclusions}

This paper presents a first branch-price-and-cut, $\mathbf{B} \& \mathbf{P} \& \mathbf{C}(\mathrm{MP})$, algorithm for solving DOMP. This approach is based on an extended formulation using an exponential number of variables coming from a set partitioning model. Elements in the partitions are couples containing information about a client and its sorted position in the sorted sequence of allocation costs. To address the solution of this formulation we develop a column generation algorithm and we prove that the pricing routine is polynomially solvable by a dynamic programming algorithm. We embed the column generation algorithm within a branch-and-price framework. Furthermore, we adapt preprocessing and incorporate families of valid inequalities that improve its performance. Extensive computational results compare the performance of our $\mathbf{B} \& \mathbf{P} \& \mathbf{C}(\mathrm{MP})$ against the most recent algorithm in the literature for DOMP, B\& $\mathbf{C}(\mathrm{WOC})$, showing that for the largest considered instances $\mathbf{B} \& \mathbf{P} \& \mathbf{C}(\mathrm{MP})$ performs better and it requires less memory to upload and run the models. The methodology presented in this paper is able to solve sized instances for DOMP that had never been solved in the literature.

\section{Acknowledgements}

This research was supported by Spanish/FEDER grants number MTM2016-74983-C02-01. The research of the second and third authors was supported by the Interuniversity Attraction Poles Programme initiated by the Belgian Science Policy Office. We thank the SCIP team (Gamrath et al. (2016)) for the helpful advices.

\section{References}

Achterberg T, Koch T, Martin A (2005) Branching rules revisited. Operations Research Letters $33: 42-54$.

Applegate D, Bixby R, Chvátal V, Cook W (1995) Finding cuts in TSP. DIMACS TR 95-05.

Barnhart C, Johnson E, Nemhauser G, Savelsbergh M, Vance P (1998) Branch-and-price: column generation for solving huge integer programs. Operations Research 46:316-329.

Beale E, Tomlin J (1970) Special facilities in a general mathematical programming system for non-convex problems using ordered sets of variables. Lawrence J, ed., Proceedings of the Fifth International Conference on Operational Research (Tavistock Publications, London).

Benichou M, Gauthier J, Girodet P, Hentges G, Ribiere G, Vincent O (1971) Experiments in mixed-integer programming. Mathematical Programming 1:71-94.

Boland N, Domínguez-Marín P, Nickel S, Puerto J (2006) Exact procedures for solving the discrete ordered median problem. Computers \& Operations Research 33(11):3270-3300.

Ceselli A, Gatto M, Lübbecke ME, Nunkesser M, Schilling H (2008) Optimizing the Cargo Express Service of Swiss Federal Railways. Transportation Science 42(4):450-465.

\footnotetext{
${ }^{1}$ For instances with $n=400$ the time limit was set to 24 hours.
} 
Chvátal V (1983) Linear Programming (W. H. Freeman and Company).

Deleplanque S, Labbé M, Ponce D, Puerto J (2018) An extended version of a Branch-Priceand-Cut Procedure for the Discrete Ordered Median Problem. Arxiv 2158317-v1.

Desrosiers J, Lübbecke M A primer in column generation. Desaulniers G, Desrosiers J, Solomon MM, eds., Column Generation, Springer, 2005.

Doulabi SHH, Rousseau LM, Pesant G (2016) A constraint-programming-based branch-andprice-and-cut approach for operating room planning and scheduling. INFORMS Journal on Computing 28(3):432-448.

du Merle O, Villenueve D, Desrosiers J, Hansen P (1999) Stabilized column generation. Discrete Mathematics 194:229-237.

Farkas G (1894) A Fourier-féle mechanikai elv alkalmazásai. Mathematikai és Természettudományi Érstesitö (12): 457-472.

Feo TA, Resende MGC (1989) A probabilistic heuristic for a computationally difficult set covering problem. Operations Research Letters 8:67-71.

Feo TA, Resende MGC (1995) Greedy ramdomized adaptive search procedures. Journal of Global Optimization 6:109-133.

Fernández E, Puerto J, Rodríguez-Chía AM (2013) On Discrete Optimization with Ordering. Annals of Operations Research 207(1): 83-96.

Fernández E, Pozo MA., Puerto J (2014) Ordered Weighted Average Combinatorial Optimization: Formulations and their properties. Discrete Applied Mathematics 169(31): 97-118.

Fernández E, Pozo MA., Puerto J, Scozzari A (2017) Ordered Weighted Average Optimization in Multiobjective Spanning Tree Problems. European Journal of Operational Research 260(31): 886-903.

Gamrath G (2010) Generic Branch-Cut-and-Price. Diploma Thesis

Gamrath G, Fischer T, Gally T, Gleixner AM, Hendel G, Koch T, Maher SJ, Miltenberger M, Müller B, Pfetsch ME, Puchert C, Rehfeldt D, Schenker S, Schwarz R, Serrano F, Shinano Y, Vigerske S, Weninger D, Winkler M, Witt JT, Witzig J (2016) The SCIP optimization suite 3.2. Technical Report 15-60, ZIB, Takustr.7, 14195 Berlin.

Günlük O, Ladányi L, de Vries S (2005) A Branch-and-Price Algorithm and New Test Problems for Spectrum Auctions. Management Science 51(3):391-406.

Johnson EL (1989) Modeling and Strong Linear Programs for Mixed Integer Programming. Wallace S, ed., Algorithms and Model Formulations in Mathematical Programming, volume 51 of NATO ASI Series, 1-43 (Springer Berlin Heidelberg), ISBN 978-3-642-83726-5.

Labbé M, Ponce D, Puerto J (2017) A comparative study of formulations and solution methods for the discrete ordered p-median problem. Computers $\&$ Operations Research 78:230 -242 .

Marín A, Nickel S, Puerto J, Velten S (2009) A flexible model and efficient solution strategies for discrete location problems. Discrete Applied Mathematics 157(5):1128-1145. 
Marín A, Nickel S, Velten S (2010) An extended covering model for flexible discrete and equity location problems. Mathematical Methods of Operations Research 71(1):125-163.

Nickel S (2001) Discrete ordered weber problems. Operations Research Proceedings 2000, 71-76 (Springer).

Nickel S, Puerto J (1999) A unified approach to network location problems. Networks 34:283290.

Nickel S, Puerto J (2005) Location Theory: A Unified Approach. (Springer).

Perea F, Puerto J (2013) Finding the nucleolus of any $n$-person cooperative game by a single linear program. Computers \& Operations Research 40(10):2308-2313.

Pessoa A, Uchoa E, Aragão MP, Rodrigues R (2010) Exact Algorithm over an ArctimeIndexed Formulation for Parallel Machine Scheduling Problems. Mathematical Programming Computation 2:259-290.

Ponce D, Puerto J, Ricca F, Scozzari A (2018) Mathematical programming formulations for the efficient solution of the $k$-sum approval voting problem. Computers $\&$ Operations Research 98:127 - 136 .

Puerto J (2008) A new formulation of the capacitated discrete ordered median problem with $\{0,1\}$-assignment. Operations Research Proceedings 2007, 165-170 (Springer), ISBN 9783-540-77902-5.

Puerto J, Fernández F (2000) Geometrical properties of the symmetrical single facility location problem. Journal of Nonlinear and Convex Analysis 1(3):321-342.

Puerto J, Pérez-Brito D, García-González CG (2014) A modified variable neighborhood search for the discrete ordered median problem. European Journal of Operational Research 234:61-76.

Puerto J, Rodríguez-Chía AM, Tamir A (2009) Minimax regret single-facility ordered median location problems on networks. INFORMS Journal on Computing 21(1):77-87.

Puerto J, Ramos AB, Rodríguez-Chía AM (2011) Single-allocation ordered median hub location problems. Computers \& Operations Research 38(2):559-570.

Puerto J, Ramos AB, Rodríguez-Chía AM (2013) A specialized Branch \& Bound \& Cut for Single-Allocation Ordered Median Hub Location problems. Discrete Applied Mathematics 161(16-17):2624-2646.

Puerto J, Ramos AB, Rodríguez-Chía AM, Sánchez-Gil MC (2016) Ordered Median Hub Location Problems with Capacity Constraints. Transportation Research Part C: Emerging Technologies 70:142-156.

Puerto J, Rodríguez-Chía AM Ordered Median Location Problems. Laporte G, Nickel S, Saldanha da Gama F, eds., Location Science, Springer, 2015.

Ryan DM, Foster A (1981) An integer programming approach to scheduling. Wren A, ed., Computer Scheduling of Public Transport: Urban Passenger Vehicle and Crew Scheduling, 269-280 (Amsterdan: North-Holland).

Wolsey LA (1998) Integer programming (New York (N.Y.), Chichester, Weinheim: J. Wiley \& sons). 